Check for updates

Cite this: RSC Adv., 2017, 7, 18120

\section{Regioselective synthesis of dipyrrolopyrazine (DPP) derivatives via metal free and metal catalyzed amination and investigation of their optical and thermal properties $\uparrow$}

\begin{abstract}
Puttavva Meti, Eun-Sil Lee, Jung-Won Yang and Young-Dae Gong (D)*
Pyrazine is an important molecular scaffold employed in organic optoelectronic materials. Here we report efficient methods for the synthesis of dipyrrolopyrazine, and pyrrolothieno-pyrazine derivatives that involve regio-selective amination reactions of dihalo-pyrrolopyrazines. The developed protocol readily affords either 2-amino- or 3-amino-pyrrolopyrazines from the corresponding 2-bromo-3-chloro-5H-pyrrolo $[2,3-b]$ pyrazines. When the amination reactions are carried under metal free under microwave irradiation, 3-amino-pyrrolopyrazines are obtained exclusively. In contrast, Buchwald cross coupling of the 2bromo-3-chloro-5H-pyrrolo[2,3-b]pyrazines affords only 2-amino-pyrrolopyrazine. The pyrrolopyrazine scaffolds were converted to the respective 1,7- and 1,5-dihydrodipyrrolo[2,3-b]pyrazines derivatives using Sonogashira reactions. A comprehensive study of the optical properties, thermal properties, and molecular packing of the synthesized compounds was carried out. The results indicate that the 1,7derivatives may be promising organic materials for optoelectronic applications.
\end{abstract}

Received 13th February 2017 Accepted 6th March 2017

DOI: 10.1039/c7ra01795b

rsc.li/rsc-advances
MW conditions proceed with enhanced regio- and stereoselectivities. ${ }^{\mathbf{1 6}}$

Benzodithiophene (BDT) is another intriguing heterocyclic scaffold that has been used as the foundation for highly efficient optoelectronic devices. ${ }^{\mathbf{1 7 2 2}}$ Although it has been shown that materials containing 2,6-diphenyl BDT analogues are good organic field effect transistors, these substances in polymers undergo rapid degradation caused by to photo-oxidation in air. ${ }^{12}$ Substitution of the benzene ring in BDT by a pyrazine moiety suppresses the photo-oxidation process. As a result, pyrazine fused dithiophenes serve as the basis for the development of new n-type transistors. Like BDT, dithienopyrazine (DTP) is planar and it undergoes molecular $\pi-\pi$ stacking in the solid state. ${ }^{23,24}$ In an effort to prepare new pyrazine fused polycyclic aromatic compounds and explore their potential use as optoelectronics materials, we have conducted an investigation exploring the synthesis of substituted dipyrrolopyrazine derivatives (DPP). At the outset, we hypothesized that the DPP derivatives would be less prone to photo-oxidation than their BDT analogues. Furthermore, it was expected that DPPs would be planar molecule in which the pyrrole ring participates in electron delocalization leading to potentially beneficial electronic properties.

In the studies described below, we developed efficient routes for the synthesis of new 2- and 3-amine functionalised pyrrolopyrazine which serve as advanced precursors to variety of new DPP derivatives. The routes for this purpose utilize key, regioselective 2- and 3-C-N functionalization reactions of 2-bromo-3- 
chloro-5H-pyrrolo[2,3-b]pyrazines 2a,b using MW irradiation (metal free conditions) and metal catalysed conditions, respectively. The products of these processes were employed to prepare 1,7- and 1,5-dihydrodipyrrolo[3,2-b:3', $\left.2^{\prime}-e\right]$ pyrazine derivatives (DPP), whose optical, thermal and morphological properties were evaluated. Finally, to assess the applicability of the DPP derivatives to optoelectronics, a systematic investigation of the photo physical and thermal properties of these substances were performed.

\section{Results and discussion}

\subsection{Synthesis and characterization}

The 2-bromo-3-chloro-5 $H$-pyrrolo[2,3-b]pyrazines 2a,b, key intermediates in the routes for synthesis of the 1,7- and 1,5dihydrodipyrrolo $\left[3,2-b: 3^{\prime}, 2^{\prime}-e\right]$ pyrazines, were prepared in two steps starting with commercially available 3,5-dibromo-6chloropyrazin-2-amine. The sequences commenced with Sonogashira cross-coupling of a dibromo-chloropyrazine using appropriate alkynes (Scheme 1). $t$-BuOK promoted intramolecular cyclization then produces $\mathbf{2 a}, \mathbf{b}$ in good yields. In addition to the 5 - $\mathrm{NH}$ substrates $3 \mathbf{c}, \mathbf{d}$, pyrrolopyrazines containing the electron donating 5 -NMe $(\mathbf{3 a}, \mathbf{b})$ and electron withdrawing 5 -NTs (3e) substituents were prepared from $\mathbf{2 a}, \mathbf{b}$ to explore the challenging $\mathrm{C}-\mathrm{N}$ coupling process.

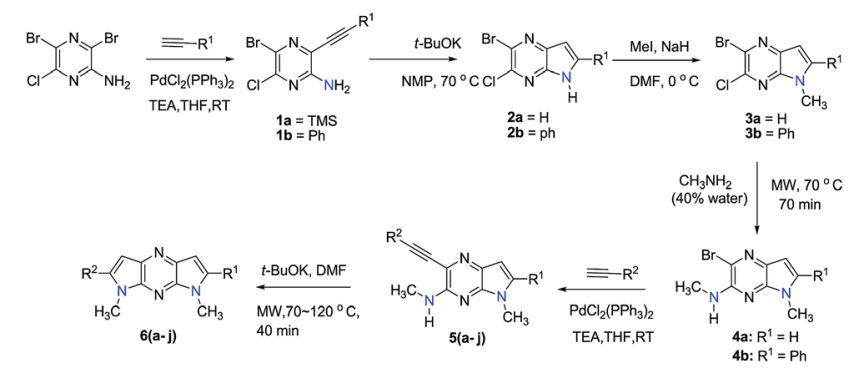

Scheme 1 Synthesis of 1,7-dihydrodipyrrolo[2,3;3'2'-e]pyrazine derivatives.
Under normal conditions ( $40 \mathrm{vol} \%$ in water, $60^{\circ} \mathrm{C}$ ), the pyrrolopyrazine derivatives $3 \mathbf{a}^{-\mathbf{e}}$ did not react with methylamine to produce the corresponding amination products. Exploratory studies showed that the use of MW conditions with THF as the solvent facilitate the $\mathrm{C}-\mathrm{N}$ bond forming substitution reactions of these pyrrolopyrazine derivatives and methylamine, although little selectivity was observed to occur between $\mathrm{C}-\mathrm{N}$ bond formation at the 2-bromo and 3-chlorosubstituted centers. Specifically, reactions of the free amines $3 \mathbf{c}, \mathbf{d}$ and $N$ tosyl derivative $3 \mathbf{e}$ react under these conditions form mixtures of 2- and 3- $\mathrm{N}$-methylamine coupling products (Table 1). Interestingly, under solvent free conditions, the $N$-Me derivatives $\mathbf{3 a}, \mathbf{b}$ react to produce exclusively the $3-\mathrm{N}$-methylamine products $\mathbf{4 a}, \mathbf{b}$ in $69 \%$ and $39 \%$ yield, respectively. The $5-N$-methylamine derivatives $\mathbf{4 a}, \mathbf{b}$, formed in the manner described above were then used to prepare the 1,7-dihydrodipyrrolo[2,3-b;3' $\left.2^{\prime}-e\right]$ pyrazines $\mathbf{6 a}-\mathbf{j}$. To this end, $\operatorname{Pd}\left(\mathrm{PPh}_{3}\right)_{2} \mathrm{Cl}_{2}$ catalysed Sonogashira reaction of $\mathbf{4 a}, \mathbf{b}$ with a series of terminal alkynes were carried out to generate the respective 2-ethynyl- $\mathrm{N}, 5$-dimethyl-5 $\mathrm{H}$-pyrrolo [2,3-b]pyrazin-3-amines 5a-j (59-74\%, Table 2). Finally, $t$-BuOK mediated intramolecular cyclization of $\mathbf{5 a} \mathbf{a} \mathbf{j}$ form the corresponding target 1,7-dihydrodipyrrolo[2,3-b:3 $\left.3^{\prime}, 2^{\prime}-e\right]$ pyrazines $\mathbf{6 a - j}$ in good to excellent yields (63-96\%).

Our attention was next focussed at the synthesis of the 1,5dihydrodipyrrolo $\left[3,2-b: 3^{\prime}, 2^{\prime}-e\right]$ pyrazine derivatives. For this purpose, $3 \mathbf{a}, \mathbf{b}$ were subjected to reactions using the standard Buchwald coupling conditions. $\mathrm{Pd}(\mathrm{OAc})_{2}$ with xantphos as the ligand was selected as the catalyst together with $t$-butyl carbamate as the coupling partner in the process. ${ }^{25}$ As expected, these reactions takes place exclusively at the 2-bromo positions in $\mathbf{3 a}, \mathbf{b}$ to give the respective $2-\mathrm{N}$-Boc protected derivatives $\mathbf{7 a}, \mathbf{b}$ in good yields. Subsequent $N$-methylation of $7 \mathbf{a}, \mathbf{b}$ followed by Boc removal using TFA produces the corresponding $2-\mathrm{N}$-methylamine derivatives $\mathbf{9 a , b}$. Sonogashira cross coupling reactions of 9a,b with a variety of different terminal alkynes were investigated. In accordance with the earlier observations, under nonMW conditions the starting materials remained unreactive. Fortunately, under MW (Scheme 2), the desired products 10a-k

Table 1 The influence of $5-N$-substituents on $\mathrm{C}-\mathrm{N}$ coupling reactions of pyrrolopyrazines ${ }^{a}$

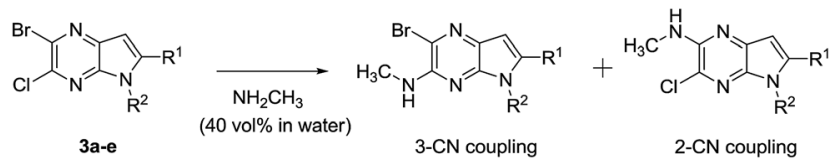

\begin{tabular}{|c|c|c|c|c|c|}
\hline Compound & $\mathrm{R}^{1} / \mathrm{R}^{2}$ & Solvent & Temp. $\left({ }^{\circ} \mathrm{C}\right)$ & Time (min) & $3-\mathrm{CN}: 2-\mathrm{CN}^{b}$ \\
\hline $3 a$ & $\mathrm{H} / \mathrm{Me}$ & Neat & 60 & 70 & $100: 0(69 \%)^{c}$ \\
\hline $3 \mathbf{b}$ & $\mathrm{Ph} / \mathrm{Me}$ & Neat & 60 & 70 & $100: 0(39 \%)^{c}$ \\
\hline $3 c$ & $\mathrm{Ph} / \mathrm{H}$ & Neat & 65 & 70 & Mixture \\
\hline $3 d$ & $\mathrm{H} / \mathrm{H}$ & Neat & 65 & 70 & $50: 50$ \\
\hline $3 e$ & $\mathrm{H} / \mathrm{Ts}$ & Neat & 60 & 50 & $20: 30^{d}$ \\
\hline
\end{tabular}

${ }^{a}$ All reactions were carried out under microwave (MW) conditions. ${ }^{b}$ LC/MS analysis. ${ }^{c}$ Isolated yields. ${ }^{d}$ About $50 \%$ starting material was detected by LC/MS analysis, in addition to the product of its detosylation. 
Table 2 Substrate scope for preparation 1,7-dihydrodipyrrolo[3,2$b: 3^{\prime}, 2^{\prime}-$ e]pyrazine derivatives

\begin{tabular}{|c|c|c|c|c|c|c|c|}
\hline No & $\mathrm{R}^{1}$ & $\mathrm{R}^{2}$ & Yield (\%) & No & $\mathrm{R}^{1}$ & $\mathrm{R}^{2}$ & Yield (\%) \\
\hline $5 a$ & $\mathrm{H}-\{-$ & TMS & 66 & $6 a$ & $H \cdot \xi-$ & $\mathrm{H}-$ & $78^{b}$ \\
\hline $5 \mathbf{b}$ & $\mathrm{H}-\{-$ & & 68 & $6 \mathbf{b}$ & $H-\xi-$ & & 96 \\
\hline $5 c$ & $H-\xi-$ & & 65 & $6 c$ & $H-\{-$ & & 90 \\
\hline $5 d$ & $\mathrm{H}-\xi-$ & & 69 & 6d & $\mathrm{H}-\{-$ & & 88 \\
\hline $5 e$ & $\mathrm{H}-\xi_{-}$ & & 70 & $6 e$ & $\mathrm{H}-\{-$ & & 92 \\
\hline $5 f$ & $H-\{-$ & & 71 & $6 f$ & $\mathrm{H}-\{-$ & & 89 \\
\hline $5 g$ & $H-\xi-$ & & 74 & $6 \mathrm{~g}$ & $\mathrm{H}-\xi-$ & & 91 \\
\hline $5 \mathbf{h}$ & $\mathrm{H}-\{-$ & & $59^{a}$ & $6 h$ & $\mathrm{H}-\{-$ & & 76 \\
\hline $5 \mathbf{i}$ & 使等 & & 67 & $6 i$ & & & 63 \\
\hline & C & & $64^{a}$ & $6 j$ & & & 74 \\
\hline
\end{tabular}

${ }^{a}$ Trace amounts of cyclization product was observed. ${ }^{b}$ The TMS group was displaced during the reaction due to high basicity of the $t$-BuOK.

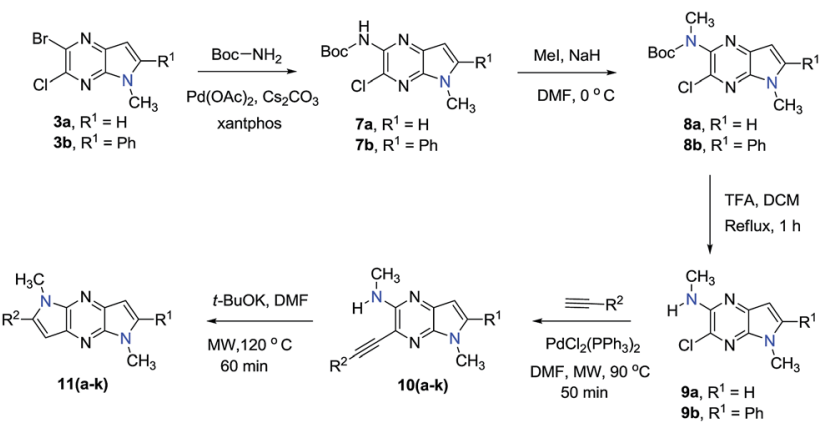

Scheme 2 Synthesis of 1,5-dihydrodipyrrolo[2,3-b;3'2'-e]pyrazine derivative.

were detected and could be isolated in average to good yields were detected and could be isolated in average to good yields (41-82\%) from 9a,b. Attempts at preparation of the target 1,5dihydrodipyrrolo $\left[3,2-b: 3^{\prime}, 2^{\prime}-e\right]$ pyrazine derivatives from $\mathbf{1 0 a}-\mathbf{k}$ by using intramolecular cyclization under non-MW conditions proved to be futile. However, under optimized condition developed above (MW irradiation for $50 \mathrm{~min}$ at $120{ }^{\circ} \mathrm{C}$ ), the corresponding 1,5-DPP 11a-k are obtained in excellent yields (Table 3).

The structures of the 1,7- and 1,5-dihydrodipyrrolo[3,2$\left.b: 3^{\prime}, 2^{\prime}-e\right]$ pyrazines, synthesized using the sequences described above, were confirmed by using NMR spectroscopy, LCMS and HRMS analysis (see ESI $\dagger$ ). It is noteworthy that the NMR spectra of $\mathbf{6 a}-\mathbf{j}$ and 11a-f exhibit similar features.

Finally, the pyrrolo[3,2-e]thieno[2,3-b]pyrazines 13a,b were prepared by using the methodology developed in the effort
Table 3 Substrate scope for preparation of 1,5-dihydrodipyrrolo[3,2$\left.b: 3^{\prime}, 2^{\prime}-e\right]$ pyrazine derivatives

\begin{tabular}{|c|c|c|c|c|c|c|c|}
\hline No & $\mathrm{R}^{1}$ & $\mathrm{R}^{2}$ & Yield (\%) & No & $\mathrm{R}^{1}$ & $\mathrm{R}^{2}$ & Yield (\%) \\
\hline $10 a$ & $\mathrm{H} \cdot \xi_{-}$ & TMS-\{- & $53^{a}$ & $11 \mathrm{a}$ & $\mathrm{H}-\{-$ & $\mathrm{H}-\hat{\xi}-$ & $87^{b}$ \\
\hline $10 \mathrm{~b}$ & $\mathrm{H} \cdot \hat{\xi}-$ & & $62^{a}$ & $11 b$ & $\mathrm{H}-\{-$ & & 91 \\
\hline $10 c$ & $\mathrm{H}-\{-$ & & 41 & $11 \mathrm{c}$ & $\mathrm{H}-\{-$ & & 84 \\
\hline 10d & $\mathrm{H}-\xi^{-}$ & & $52^{a}$ & 11d & $\mathrm{H}-\{-$ & & 78 \\
\hline $10 e$ & $\mathrm{H}-\{$ & & $61^{a}$ & $11 \mathrm{e}$ & $1-\{-$ & & 82 \\
\hline $10 f$ & & & 68 & $11 \mathrm{f}$ & & & 92 \\
\hline $10 \mathrm{~g}$ & & & 74 & $11 \mathrm{~g}$ & & & 90 \\
\hline $10 h$ & & & 78 & $11 \mathrm{~h}$ & & & 90 \\
\hline $10 \mathrm{i}$ & & & 66 & $11 \mathrm{i}$ & & & 83 \\
\hline $10 \mathrm{j}$ & & & 69 & $11 \mathrm{j}$ & & & 85 \\
\hline $10 k$ & & 73 & 82 & $11 k$ & & & 91 \\
\hline
\end{tabular}

${ }^{a}$ Trace amount of cyclization product was also observed. ${ }^{b}$ TMS group was displaced during reaction due to high basicity of the reagent.

described above. Accordingly, palladium catalysed reactions of 3a,b and phenyl acetylene followed by intramolecular cyclization $^{26}$ gives 13a,b in moderate yields (Scheme 3).

In an attempt to develop a more efficient routes for synthesis of the highly interesting symmetric 1,5-dipyrrolopyrazines, the double cyclization strategy was depicted in Scheme 4 was explored. For this purpose, symmetric 2,5-dibromo-3,6-dichloro pyrazine (16) was prepared using literature procedures and subjected to a double Sonogashira coupling process. The bisacetylene derivative $\mathbf{1 7}$ was produced in only low yield along with unreacted starting material in this reaction. Next, displacement of both chlorides in $\mathbf{1 7}$ with methyl amine groups was examined. However, only the mono-aminated product 18 is produced. Therefore this route was abandoned in favour of the aforementioned protocol.

The solubility and stabilities of the dipyrrolopyrazine derivatives in diverse organic solvents is an important criteria for their applications in optoelectronic devices. Therefore, the solubilities of these crystalline substances were assessed. All of the tested dipyrrolopyrazine proved to be highly soluble in

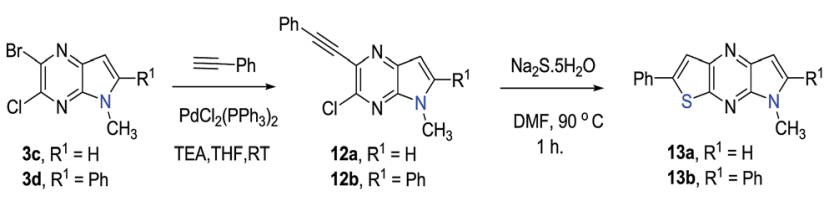

Scheme 3 Annulation sequence for synthesis of pyrrolo[3,2-e]thieno $[2,3-b]$ pyrazine. 


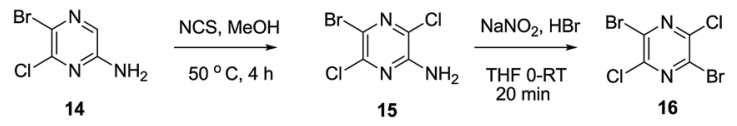

14<smiles>CC#Cc1nc(NC)c(C#CC)nc1NC</smiles>

Not observed

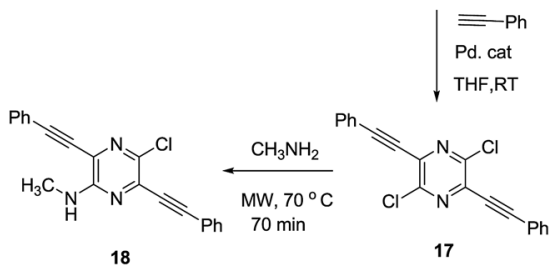

18
Scheme 4 Double cyclization approach to prepare 1,5-dihydrodipyrrolo[3,2-b:3', $\left.2^{\prime}-e\right]$ pyrazine. a)

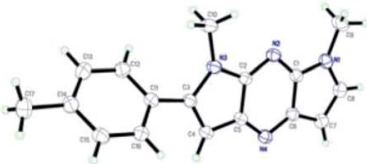

b)

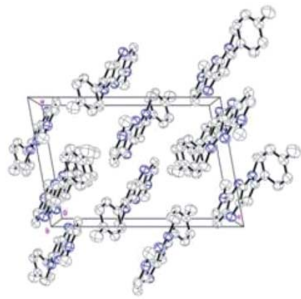

Fig. 1 (a) Crystal structure of 6f. (b) Packing diagram of $6 \mathrm{f}$ viewed along $b$ axis in a bulk single crystal.

$\mathrm{CH}_{2} \mathrm{Cl}_{2}, \mathrm{MeOH}$, THF and $\mathrm{CHCl}_{3}$. Additionally, all of the crystalline in nature. Pyrrolopyrazines have long shelf lives, ranging up to one year at room temperature. Next, a comparative study of the optical and thermal properties of the three different pyrazine cores was undertaken.

\subsection{Crystallographic analysis}

A single crystal of pyrrolopyrazine 6 f was grown by slow evaporation of a mixture of ether and dichloromethane. X-ray crystallographic analysis revealed that 6 f forms monoclinic crystal system with space group $P 2(1) / n$ and is planar with the exception of slight twist of the phenyl substituent. Furthermore, possibly as a consequence of its planar backbone, pyrrolopyrazine derivative $\mathbf{6 f}$ adopt a near perfect orthogonal orientation in the crystalline state ${ }^{27}$ (Fig. 1).

\section{Photophysical properties}

\subsection{Thermal properties}

The thermal properties of one member of each of the three types of pyrazine derivative prepared above (6i, 11f, and 13b) were subjected to thermogravimetric analysis and differential scanning calorimetry (Fig. 2). The phase transitions of the selected compounds were analysed using DSC under a nitrogen atmosphere with a heating rate of $10{ }^{\circ} \mathrm{C} \mathrm{min}^{-1}$. The results of both the TGA and DSC analyses reveal that all of these substances possess high thermal stabilities. A sharp endothermic melting peak was observed in each case, which confirms the highly crystalline nature of the compounds (Table 5). Furthermore, the
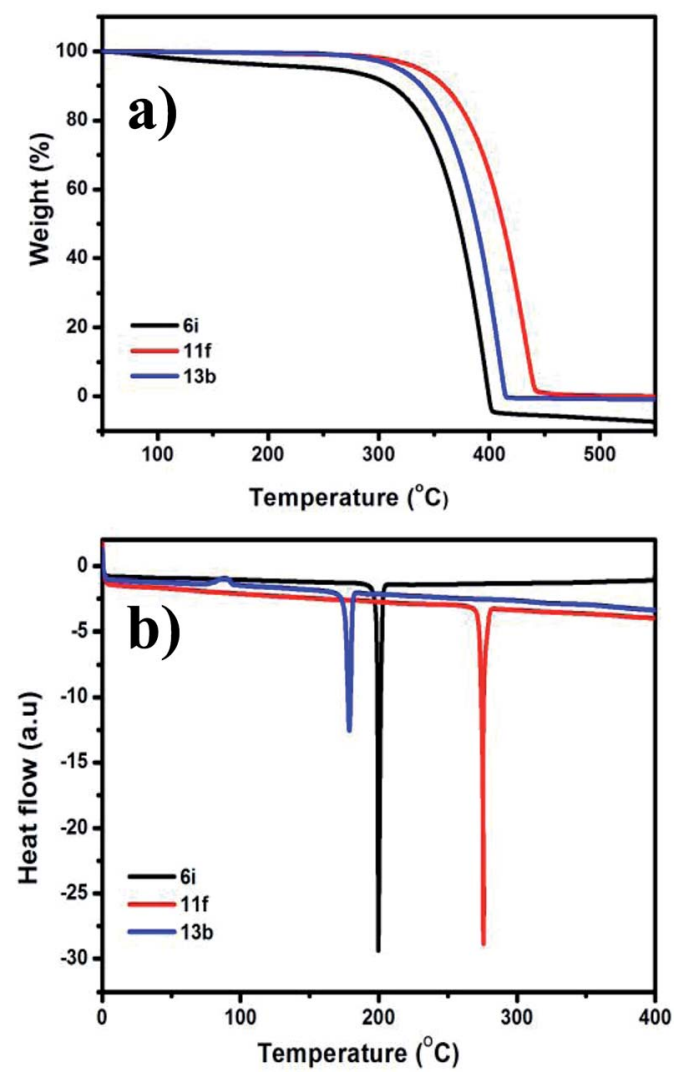

Fig. 2 (a) TGA and (b) DSC thermograms of the 6i, 11f, and 13b recorded with a heating rate of $10{ }^{\circ} \mathrm{C} \min ^{-1}$ under nitrogen atmosphere.

high $T_{\mathrm{d}}$ and $T_{\mathrm{m}}$ of $\mathbf{6 i}, \mathbf{1 1 f}$, and $\mathbf{1 3 b}$ indicate that they have remarkable thermal stabilities.

\subsection{Optical properties}

The optical properties of the prepared pyrazine derivatives in dichloromethane were evaluated by using absorption and emission spectroscopy (Fig. 3). The results show that the presence of the phenyl substituent (i.e., in $\mathbf{6 i}, \mathbf{j})$ and acetylene (5a-j) groups on the pyrrolopyrazine core result in a significant bathochromic shift with respect to those of unsubstituted pyrrolopyrazine derivatives. The extended conjugation in the substituted compounds is the likely reasons for the observed bathochromic shift. The absorption maxima $\left(\lambda_{\max }\right)$ occur between 386-410 $\mathrm{nm}$ for the acyclic compounds $\mathbf{5 a}-\mathbf{j}$ and at 358-380 nm for the cyclic analogues $\mathbf{6 a}-\mathbf{j}$, respectively. A similar trend is observed for the 1,5-dihydrodipyrrolo[3,2-b:3', $\left.2^{\prime}-e\right]$ pyrazine derivatives 11a-k (Table 4). Substitution at positions 2 and 6 of the DPP core (i.e., 11i,g) leads to a longer conjugation length, thereby resulting in red shift of the absorption maximum. The absorption spectrum of $\mathbf{6 h}$ is especially remarkable in that it displays an unusually large bathochromic shift, presumably as a result of intramolecular charge transfer excitation (ICT), in addition to a localised $\pi-\pi^{*}$ transition as observed in Fig. 3(b). Comparisons of the results of the 

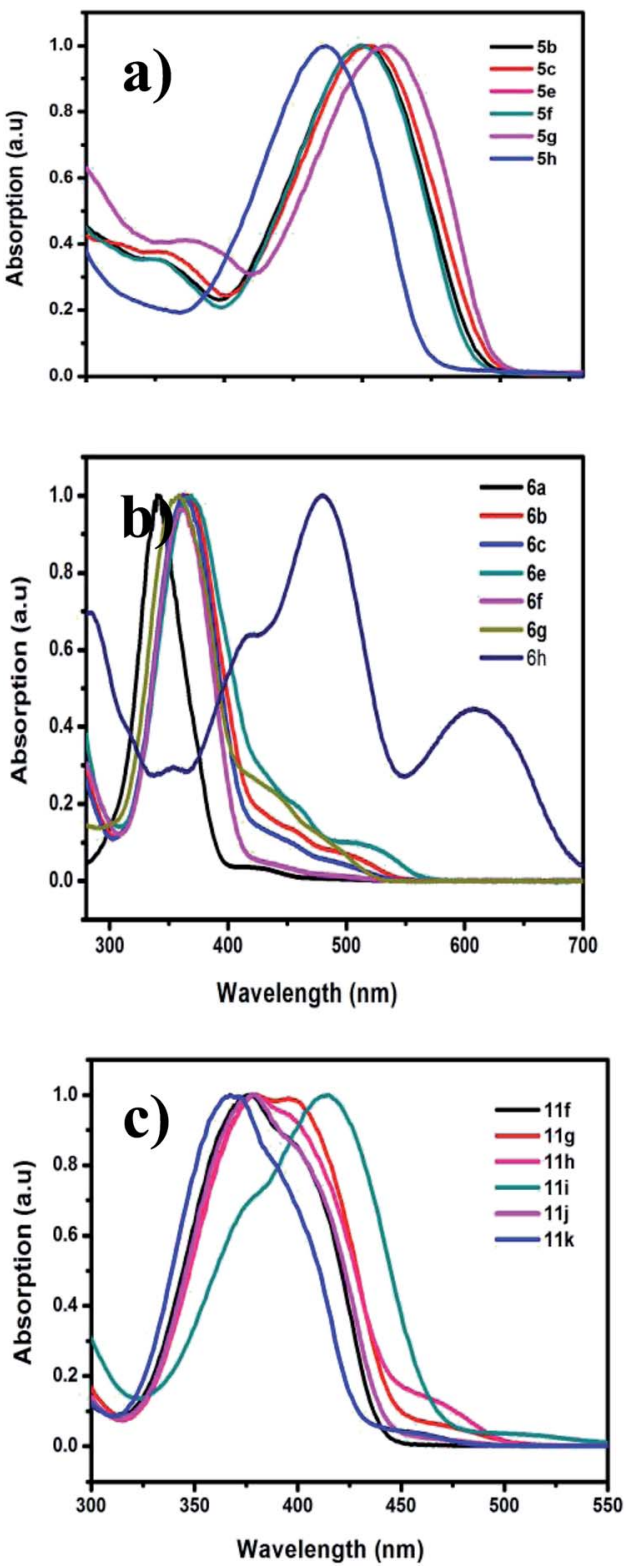

Fig. 3 UV-vis absorption spectra of DPP derivatives in DCM. (a) Acyclic compounds 5, (b) cyclic compounds 6, (c) cyclic 2,6-substituted DPP series 11

Table 4 UV-visible spectroscopic data of DPP derivatives

\begin{tabular}{llllll}
\hline Molecules & $\lambda_{\max }{ }^{a}(\mathrm{~nm})$ & $E_{\mathrm{g}}^{\mathrm{opt} b}(\mathrm{eV})$ & Molecules & $\lambda_{\max }{ }^{a}(\mathrm{~nm})$ & $E_{\mathrm{g}}^{\mathrm{opt} b}(\mathrm{eV})$ \\
\hline $\mathbf{5 b}$ & 400 & 2.76 & $\mathbf{6 f}$ & 366 & 2.81 \\
$\mathbf{5 c}$ & 402 & 2.73 & $\mathbf{6 g}$ & 479,613 & 1.75 \\
$\mathbf{5 e}$ & 400 & 2.76 & $\mathbf{6 h}$ & 358 & 2.65 \\
$\mathbf{5 f}$ & 400 & 2.76 & $\mathbf{1 1 f}$ & 378 & 2.76 \\
$\mathbf{5 g}$ & 410 & 2.72 & $\mathbf{1 1 g}$ & 385 & 2.66 \\
$\mathbf{5 h}$ & 386 & 2.88 & $\mathbf{1 1 h}$ & 381 & 2.61 \\
$\mathbf{6 b}$ & 360 & 2.81 & $\mathbf{1 1 i}$ & 413 & 2.66 \\
$\mathbf{6 c}$ & 360 & 2.96 & $\mathbf{1 1 j}$ & 380 & 2.78 \\
$\mathbf{6 e}$ & 367 & 2.66 & $\mathbf{1 1 k}$ & 367 & 2.81
\end{tabular}

${ }^{a}$ Absorption spectra measured in DCM solvent. ${ }^{b}$ Optical band gap calculated from the UV-vis absorption onset in solution.
Table 5 Optical and thermal properties of selected scaffolds

\begin{tabular}{llllll}
\hline Molecules & $\lambda_{\max }{ }^{a}(\mathrm{~nm})$ & $\lambda_{\max }{ }^{b}(\mathrm{~nm})$ & $E_{\mathrm{g}}^{\mathrm{optc}}(\mathrm{eV})$ & $T_{\mathrm{d}}{ }^{d}\left({ }^{\circ} \mathrm{C}\right)$ & $T_{\mathrm{m}}{ }^{e}\left({ }^{\circ} \mathrm{C}\right)$ \\
\hline 6i & 378 & 443 & 2.70 & 298 & 200 \\
11f & 377 & 450 & 2.78 & 342 & 280 \\
13b & 384 & 446 & 2.94 & 340 & 184
\end{tabular}

${ }^{a}$ Absorption spectra. ${ }^{b}$ Emission spectra both measured in DCM solvent. ${ }^{c}$ Optical band gap calculated from the UV-vis absorption onset in solution. ${ }^{d}$ Degradation temperature observed from TGA corresponding to $5 \%$ weight loss at $10{ }^{\circ} \mathrm{C} \mathrm{min}^{-1}$ under a nitrogen atmosphere. ${ }^{e}$ Melting temperature observed from DSC at $10{ }^{\circ} \mathrm{C}$ $\min ^{-1}$ under nitrogen atmosphere.
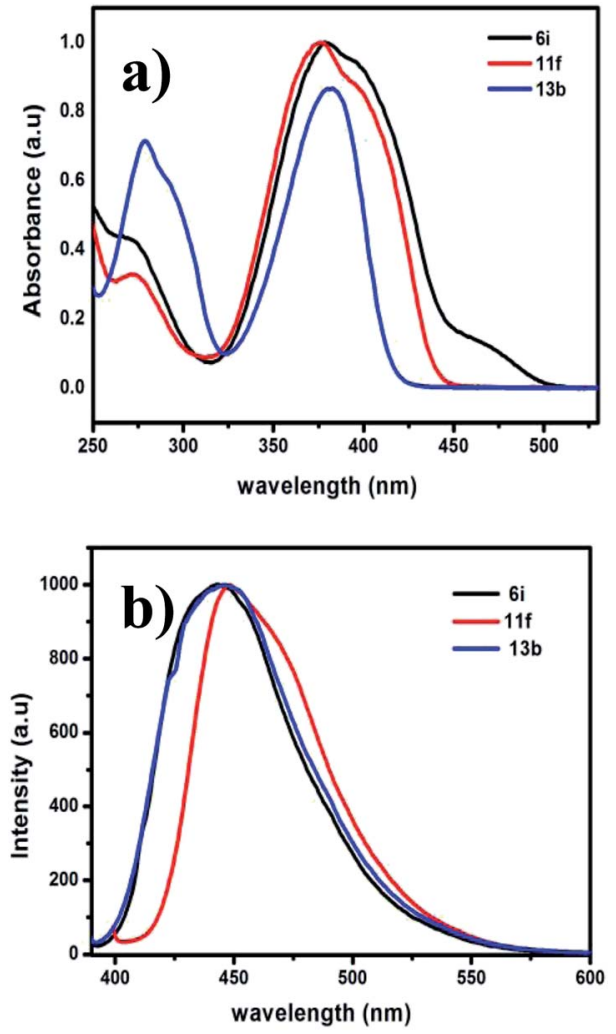

Fig. 4 (a) UV-vis absorption spectra of $6 \mathrm{i}, 11 \mathrm{f}$, and $13 \mathrm{~b}$ in DCM. (b) Emission spectra of $6 \mathrm{i}, 11 \mathrm{f}$, and $13 \mathrm{~b}$ in DCM when excited at $375 \mathrm{~nm}$.

spectroscopy studies reveal that $\mathbf{6 i}$ and $\mathbf{1 3 b}$ have similar absorption and emission properties (Table 5). Interestingly, 11f shows a significant red shift in its absorption spectrum (Fig. 4). The optical band gap energies $\left(E_{\mathrm{g}}\right)$ for $\mathbf{6 i}, \mathbf{1 1 f}$, and 13b approximated using the onset of their absorption bands, are 2.7, 2.78, and $2.94 \mathrm{eV}$, respectively.

\subsection{Structural and morphological characteristics}

The structural and morphological properties of $\mathbf{6 i}, \mathbf{1 1 f}$, and $\mathbf{1 3 b}$ were evaluated by using X-ray diffraction analysis. Each molecule displays a series of sharply resolved diffraction peaks, indicating that all the compounds possess a high degree of ordering in the solid state (Fig. 5). 


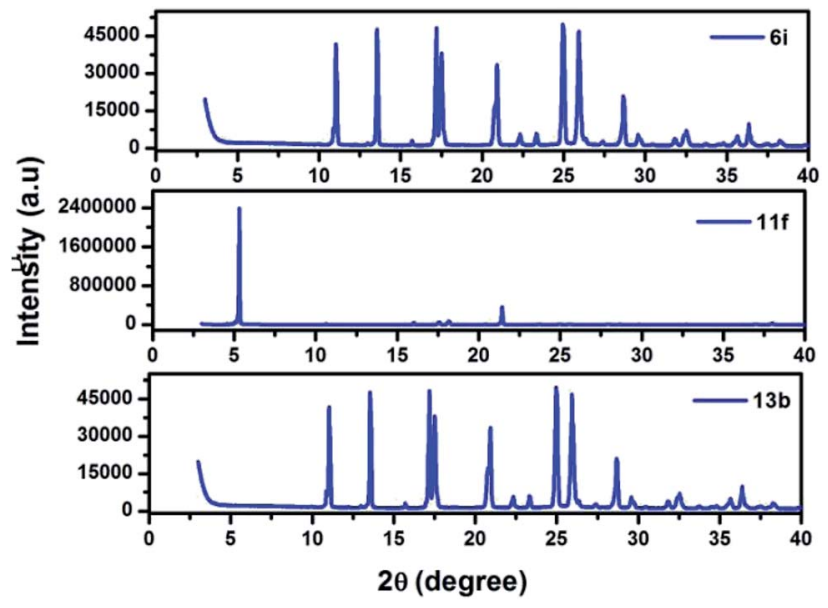

Fig. 5 Powder X-ray diffraction patterns of $6 \mathrm{i}, 11 \mathrm{f}$, and $13 \mathrm{~b}$ at room temperature.

\section{Experimental section}

\subsection{General method and materials}

All chemicals and solvents are of reagent grade unless otherwise indicated. Reactions with air sensitive materials were carried out under a nitrogen atmosphere. Melting points are uncorrected. ${ }^{1} \mathrm{H}$ NMR and ${ }^{13} \mathrm{C}$ NMR spectra were recorded using a Bruker $500 \mathrm{MHz}$ NMR spectrometer and are given in ppm $(\delta)$ units relative to protons and carbons in the deuterated solvent. Mass spectra were recorded using 6400 Triple Quadrupole LC/ MS (Agilent). High-resolution mass spectrometry was performed using a $6550 \mathrm{iFunnel}$ Q-TOF LC/MS system (Agilent). UV-vis spectra were recorded using Varian cary-50 spectrophotometer. TGA and DSC were performed with TGA 3 plus and DSC 2 STAR system, respectively. X-ray diffraction and single crystal XRD analysis was performed using a Smartlab and Bruker D8 Discover X-ray Diffractometer with GADDS, respectively.

5-Bromo-6-chloro-3-((trimethylsilyl)ethynyl)pyrazin-2-amine (1a). To a stirred solution of 3,5-dibromo-6-chloro-pyrazin-2ylamine $(2.0 \mathrm{~g}, 7.04 \mathrm{mmol})$ in anhydrous THF $(20 \mathrm{~mL})$ at $0{ }^{\circ} \mathrm{C}$, TEA $(2.1 \mathrm{~g}, 21 \mathrm{mmol})$ and CuI $(0.13 \mathrm{~g}, 0.7 \mathrm{mmol})$ were added subsequently. The mixture was purged thoroughly with nitrogen for $20 \mathrm{~min}$ followed by the addition of $\mathrm{Pd}\left(\mathrm{PPh}_{3}\right)_{2} \mathrm{Cl}_{2}$ (0.49 g, $0.7 \mathrm{mmol})$. Next, TMS-acetylene $(1.0 \mathrm{~mL}, 7.04 \mathrm{mmol})$ was added drop wise and the mixture was allowed to slowly warm to $15^{\circ} \mathrm{C}$ over a period of $3 \mathrm{~h}$. Then the mixture was diluted with water and extracted with EtOAc $(3 \times)$. The combined organic extracts were concentrated in vacuum and the residue was subjected to silica gel column chromatography to give title compound as a yellow solid. Yield: $81 \%$. The NMR data was found to be identical to that reported in literature. Yield: $81 \%$, LC-MS (ESI): $m / z=306.10[\mathrm{M}+2]^{+}$.

5-Bromo-6-chloro-3-(phenylethynyl)pyrazin-2-amine The above procedure was followed and the reaction mixture is stirred at room temperature for $2 \mathrm{~h}$. Yield: $83 \%,{ }^{1} \mathrm{H}$ NMR (500 $\left.\mathrm{MHz}, \mathrm{CDCl}_{3}\right) \delta 7.63-7.55(\mathrm{~m}, 2 \mathrm{H}), 7.50-7.38(\mathrm{~m}, 3 \mathrm{H}), 5.27(\mathrm{~s}$, 2H). ${ }^{13} \mathrm{C}$ NMR $\left(126 \mathrm{MHz}, \mathrm{CDCl}_{3}\right) \delta 153.3,145.9,132.0,129.9$,
128.6, 123.9, 122.4, 120.9, 98.0, 82.4. LC-MS (ESI): $\mathrm{m} / z=310.1$ $[\mathrm{M}+2]^{+}$. HRMS (ESI) $m / z:[\mathrm{M}+\mathrm{H}]^{+}$calculated for $\mathrm{C}_{12} \mathrm{H}_{7} \mathrm{BrClN}_{3}$, 307.9585; found 307.9606.

2-Bromo-3-chloro-5H-pyrrolo[2,3-b]pyrazine (2a). To a solution of 5-bromo-6-chloro-3-((trimethylsilanyl)ethynyl)pyrazin-2amine $(1.0 \mathrm{~g}, 3.2 \mathrm{mmol})$ in anhydrous NMP $(20 \mathrm{~mL})$ a solution of $t$-BuOK in NMP $(0.7 \mathrm{~g}, 6.5 \mathrm{mmol})$ was added slowly under a nitrogen atmosphere. The mixture was refluxed at $80{ }^{\circ} \mathrm{C}$ for $1 \mathrm{~h}$, cooled to ambient temperature, and diluted with EtOAc and water. The organic layer was separated, dried over $\mathrm{MgSO}_{4}$, concentrated to give a light brown solid. This material was used directly without further purification. Yield: $72 \%,{ }^{1} \mathrm{H}$ NMR (500 MHz, DMSO) $\delta 12.52(\mathrm{~s}, 1 \mathrm{H}), 8.1$ (d, J=1.8 Hz, 1H), 6.70 (d, $J=1.8 \mathrm{~Hz}, 1 \mathrm{H}) .{ }^{13} \mathrm{C}$ NMR (126 MHz, DMSO) $\delta$ 139.1, 139.1, 138.5, 134.4, 129.9, 100.7. LC-MS (ESI): $m / z=234.0[\mathrm{M}+2]^{+}$.

2-Bromo-3-chloro-6-phenyl-5H-pyrrolo[2,3-b]pyrazine (2b). Yield: 78\%, ${ }^{1} \mathrm{H}$ NMR (500 MHz, DMSO) $\delta 12.97$ (s, 1H), 8.02 (d, $J$ $=7.7 \mathrm{~Hz}, 2 \mathrm{H}), 7.55(\mathrm{t}, J=7.5 \mathrm{~Hz}, 2 \mathrm{H}), 7.48(\mathrm{t}, J=7.2 \mathrm{~Hz}, 1 \mathrm{H})$, 7.24 (s, 1H). ${ }^{13} \mathrm{C}$ NMR (126 MHz, DMSO) $\delta$ 145.8, 140.8, 139.6, 139.0, 130.5, 130.3, 130.3, 129.6, 126.5, 97.6. LC-MS (ESI): $m / z=$ $310.0[\mathrm{M}+2]^{+}$. HRMS (ESI) $\mathrm{m} / \mathrm{z}:[\mathrm{M}+\mathrm{H}]^{+}$calculated for $\mathrm{C}_{12} \mathrm{H}_{7^{-}}$ $\mathrm{BrClN}_{3}, 307.9585$; found 307.9604 .

2-Bromo-3-chloro-5-methyl-5H-pyrrolo[2,3-b]pyrazine (3a). To a solution of 2-bromo-3-chloro-5 $H$-pyrrolo[2,3- $b]$ pyrazine $(3.0 \mathrm{~g}, 12.9 \mathrm{mmol})$ in DMF $(40 \mathrm{~mL})$ at $0{ }^{\circ} \mathrm{C}, \mathrm{NaH}$ (60\% dispersion in mineral oil, $0.36 \mathrm{~g}, 12.9 \mathrm{mmol}$ ) was added carefully under nitrogen atmosphere. After stirring for $30 \mathrm{~min}$, MeI $(0.8 \mathrm{~mL}$, $12.9 \mathrm{mmol}$ ) was added and the mixture was warmed slowly to room temperature. After $3 \mathrm{~h}$, the mixture is poured into icecoldwater and the precipitate was collected by vacuum filtration. The crude solid is dissolved in EtOAc and subjected to silica gel chromatography to give title compound. (20\% EA : hexane). Yield: $90 \%,{ }^{1} \mathrm{H}$ NMR $\left(500 \mathrm{MHz} \mathrm{CDCl}_{3}\right) \delta 7.46(\mathrm{~d}, J$ $=3.6 \mathrm{~Hz}, 1 \mathrm{H}), 6.65(\mathrm{~d}, J=3.5 \mathrm{~Hz}, 1 \mathrm{H}), 3.88(\mathrm{~s}, 3 \mathrm{H}) .{ }^{13} \mathrm{C}$ NMR $(126$ $\left.\mathrm{MHz} \mathrm{CDCl}_{3}\right) \delta 140.6,138.6,138.0,134.7,131.0,100.6,31.8$. LCMS (ESI): $m / z=248.0[\mathrm{M}+2]^{+}$. HRMS (ESI) $m / z:[\mathrm{M}+\mathrm{H}]^{+}$calcd for $\mathrm{C}_{7} \mathrm{H}_{5} \mathrm{BrClN}_{3}, 245.9428$; found 245.9436 .

2-Bromo-3-chloro-5-methyl-6-phenyl-5H-pyrrolo[2,3-b]pyrazine (3b). Yield: $88 \%,{ }^{1} \mathrm{H}$ NMR $\left(500 \mathrm{MHz}, \mathrm{CDCl}_{3}\right) \delta 7.55$ (dd, $J=$ 8.3, 4.4 Hz, 5H), 6.70 (s, 1H), 3.86 (s, 3H). ${ }^{13} \mathrm{C}$ NMR $(126 \mathrm{MHz}$, $\left.\mathrm{CDCl}_{3}\right) \delta 147.9,140.2,140.0,138.2,131.2,130.6,129.6,129.1$, 128.9, 100.1, 30.2. LC-MS (ESI): $m / z=324.0[\mathrm{M}+2]^{+}$. HRMS (ESI) $m / z:[\mathrm{M}+\mathrm{H}]^{+}$calculated for $\mathrm{C}_{13} \mathrm{H}_{9} \mathrm{BrClN}_{3}, 321.9741$; found 321.9762 .

2-Bromo- $\mathrm{N}, 5$-dimethyl-5H-pyrrolo[2,3-b]pyrazin-3-amine (4a). To 2-bromo-3-chloro-5-methyl-5 $H$-pyrrolo[2,3-b]pyrazine $(0.1 \mathrm{~g}$, $0.4 \mathrm{mmol}$ ) is added methylamine ( $2 \mathrm{~mL}, 40 \%$ solution in water). The mixture was stirred at reflux for $40 \mathrm{~min}$ in a microwave vial. After cooling, the mixture was extracted with EtOAc. The organic layer was washed with water and dried over $\mathrm{MgSO}_{4}$, and concentrated in vacuum giving a residue that was subjected to silica gel chromatography to give desired product. Yield: $69 \%$, ${ }^{1} \mathrm{H}$ NMR $\left(500 \mathrm{MHz}, \mathrm{CDCl}_{3}\right) \delta 6.99(\mathrm{~d}, J=3.6 \mathrm{~Hz}, 1 \mathrm{H}), 6.43(\mathrm{~d}, J=$ $3.6 \mathrm{~Hz}, 1 \mathrm{H}), 5.13(\mathrm{~s}, 1 \mathrm{H}), 3.75(\mathrm{~s}, 3 \mathrm{H}), 3.07$ (d, $J=5.0 \mathrm{~Hz}, 3 \mathrm{H}) .{ }^{13} \mathrm{C}$ NMR (126 MHz, $\left.\mathrm{CDCl}_{3}\right) \delta 147.6,139.3,129.5,127.0,121.4,100.1$, 31.1, 28.7. LC-MS (ESI): $m / z=241.1[\mathrm{M}+\mathrm{H}]^{+}$. HRMS (ESI) $m / z$ : $[\mathrm{M}+\mathrm{H}]^{+}$calculated for $\mathrm{C}_{8} \mathrm{H}_{9} \mathrm{BrN}_{4}, 241.0083$; found 241.0090. 
2-Bromo- $N, 5$-dimethyl-6-phenyl-5H-pyrrolo[2,3-b]pyrazin-3amine (4b). Yield: 41\%, ${ }^{1} \mathrm{H}$ NMR $\left(500 \mathrm{MHz}, \mathrm{CDCl}_{3}\right) \delta 7.54(\mathrm{~d}, J=$ $7.6 \mathrm{~Hz}, 2 \mathrm{H}), 7.49$ (t, $J=7.3 \mathrm{~Hz}, 2 \mathrm{H}), 7.42(\mathrm{t}, J=7.1 \mathrm{~Hz}, 1 \mathrm{H}), 6.54$ (s, 1H), 5.19 (s, 1H), $3.79(\mathrm{~s}, 3 \mathrm{H}), 3.13$ (d, $J=4.4 \mathrm{~Hz}, 3 \mathrm{H}) \cdot{ }^{13} \mathrm{C}$ NMR (126 MHz, $\left.\mathrm{CDCl}_{3}\right) \delta 147.6,141.1,140.2,132.2,129.8,128.8$, 128.6, 128.1, 121.6, 100.0, 29.7, 28.7. LC-MS (ESI): $\mathrm{m} / z=317.0$ $[\mathrm{M}+\mathrm{H}]^{+}$. HRMS (ESI) $m / z:[\mathrm{M}+\mathrm{H}]^{+}$calculated for $\mathrm{C}_{14} \mathrm{H}_{13} \mathrm{BrN}_{4}$, 317.0396; found 317.0406 .

\subsection{General procedure for Sonogashira reactions}

To a stirred solution of 2-bromo- $N, 5$-dimethyl-5 $H$-pyrrolo[2,3- $b$ ] pyrazin-3-amine $(0.2 \mathrm{~g}, 0.8 \mathrm{mmol})$ in THF $(15 \mathrm{~mL})$ was added TEA (0.25 mL, $24 \mathrm{mmol}), \mathrm{Pd}\left(\mathrm{PPh}_{3}\right)_{2} \mathrm{Cl}_{2}(0.058 \mathrm{~g}, 0.08 \mathrm{mmol}), \mathrm{CuI}$ (0.015 g, $0.08 \mathrm{mmol})$ under a nitrogen atmosphere. After $10 \mathrm{~min}$, TMS-acetylene $(0.11 \mathrm{~g}, 0.8 \mathrm{mmol})$ was added dropwise. The mixture was stirred at room temperature for $1.5 \mathrm{~h}$ and concentrated to give a residue that was diluted with water and extracted with EtOAc. The organic layer was washed with water and dried over $\mathrm{MgSO}_{4}$, and concentrated in vacuum to give a residue that was subjected to silica gel chromatography to afford the desired compound.

$N, 5$-Dimethyl-2-((trimethylsilyl)ethynyl)-5 $H$-pyrrolo[2,3- $b]$ pyrazin-3-amine (5a). Mp: $126-128{ }^{\circ} \mathrm{C} .{ }^{1} \mathrm{H}$ NMR $(500 \mathrm{MHz}$, $\left.\mathrm{CDCl}_{3}\right) \delta 7.03(\mathrm{~s}, 1 \mathrm{H}), 6.45(\mathrm{~s}, 1 \mathrm{H}), 5.30(\mathrm{~s}, 1 \mathrm{H}), 3.76(\mathrm{~d}, J=8.2 \mathrm{~Hz}$, $3 \mathrm{H}), 3.11$ (d, $J=4.7 \mathrm{~Hz}, 3 \mathrm{H}), 0.32$ (s, 9H). ${ }^{13} \mathrm{C}$ NMR $(126 \mathrm{MHz}$, $\left.\mathrm{CDCl}_{3}\right) \delta 152.6,139.6,130.1,128.0,119.4,101.1,101.0,100.1$, 30.8, 28.2, 0.3. LC-MS (ESI): $m / z=259.2[\mathrm{M}+\mathrm{H}]^{+}$. HRMS (ESI) $m /$ $z:[\mathrm{M}+\mathrm{H}]^{+}$calcd for $\mathrm{C}_{13} \mathrm{H}_{18} \mathrm{~N}_{4} \mathrm{Si}, 259.1373$; found 259.1378 .

$N, 5$-Dimethyl-2-(phenylethynyl)-5H-pyrrolo[2,3-b]pyrazin-3amine (5b). Mp: $136-138{ }^{\circ} \mathrm{C} .{ }^{1} \mathrm{H}$ NMR (500 MHz, $\left.\mathrm{CDCl}_{3}\right) \delta 7.60$ (dd, $J=6.5,2.9 \mathrm{~Hz}, 2 \mathrm{H}), 7.43-7.29(\mathrm{~m}, 3 \mathrm{H}), 7.03(\mathrm{~d}, J=3.6 \mathrm{~Hz}$, $1 \mathrm{H}), 6.47(\mathrm{~d}, J=3.6 \mathrm{~Hz}, 1 \mathrm{H}), 5.35(\mathrm{~d}, J=3.8 \mathrm{~Hz}, 1 \mathrm{H}), 3.76(\mathrm{~s}, 3 \mathrm{H})$, $3.12(\mathrm{~d}, J=5.0 \mathrm{~Hz}, 3 \mathrm{H}) .{ }^{13} \mathrm{C} \mathrm{NMR}\left(126 \mathrm{CDCl}_{3}\right) \delta 152.4,139.6$, 131.7, 130.3, 128.7, 128.4, 127.9, 122.5, 119.6, 100.9, 94.3, 85.7, 30.9, 28.2. LC-MS (ESI): $m / z=263.1[\mathrm{M}+\mathrm{H}]^{+}$. HRMS (ESI) $m / z$ : $[\mathrm{M}+\mathrm{H}]^{+}$calcd for $\mathrm{C}_{16} \mathrm{H}_{14} \mathrm{~N}_{4}, 263.1291$; found 263.124.

2-((3-Fluorophenyl)ethynyl)- $N, 5$-dimethyl-5 $H$-pyrrolo[2,3- $b]$ pyrazin-3-amine (5c). Mp: $110-112{ }^{\circ} \mathrm{C} .{ }^{1} \mathrm{H}$ NMR $(500 \mathrm{MHz}$, $\left.\mathrm{CDCl}_{3}\right) \delta 7.33(\mathrm{ddd}, J=21.2,15.5,7.8 \mathrm{~Hz}, 3 \mathrm{H}), 7.09(\mathrm{~d}, J=8.3 \mathrm{~Hz}$, 1H), 7.05 (d, $J=3.5 \mathrm{~Hz}, 1 \mathrm{H}), 6.48$ (d, $J=3.5 \mathrm{~Hz}, 1 \mathrm{H}), 5.33$ (d, $J=$ $3.3 \mathrm{~Hz}, 1 \mathrm{H}), 3.76$ (s, 3H), $3.13(\mathrm{~d}, J=4.9 \mathrm{~Hz}, 3 \mathrm{H}) .{ }^{13} \mathrm{C}$ NMR $(126$ $\left.\mathrm{MHz}, \mathrm{CDCl}_{3}\right) \delta 162.3(\mathrm{~d}, J=246.8 \mathrm{~Hz}), 161.3,152.5,139.7,130.2$, $130.0(\mathrm{~d}, J=8.7 \mathrm{~Hz}), 128.3,127.6(\mathrm{~d}, J=3.0 \mathrm{~Hz}), 124.3(\mathrm{~d}, J=9.5$ $\mathrm{Hz}), 119.0,118.4,118.3,116.1,115.9,100.9,92.9$ (d, $J=3.4 \mathrm{~Hz})$, 86.6, 30.9, 28.2. LC-MS (ESI): $m / z=281.2[\mathrm{M}+\mathrm{H}]^{+}$. HRMS (ESI) $m / z:[\mathrm{M}+\mathrm{H}]^{+}$calculated for $\mathrm{C}_{16} \mathrm{H}_{13} \mathrm{FN}_{4}, 281.1197$; found 281.1202 .

2-((4-Fluorophenyl)ethynyl)- $N, 5$-dimethyl-5H-pyrrolo[2,3- $b]$ pyrazin-3-amine (5d). ${ }^{1} \mathrm{H} \mathrm{NMR}\left(500 \mathrm{MHz}, \mathrm{CDCl}_{3}\right) \delta 7.60(\mathrm{dd}, J=$ 7.6, $5.9 \mathrm{~Hz}, 2 \mathrm{H}), 7.32-7.27(\mathrm{~m}, 1 \mathrm{H}), 7.08(\mathrm{t}, J=8.4 \mathrm{~Hz}, 2 \mathrm{H}), 6.42$ $(\mathrm{d}, J=3.0 \mathrm{~Hz}, 1 \mathrm{H}), 5.21(\mathrm{~s}, 1 \mathrm{H}), 3.82(\mathrm{~s}, 3 \mathrm{H}), 3.12(\mathrm{~d}, J=4.9 \mathrm{~Hz}$, $3 \mathrm{H}) .{ }^{13} \mathrm{C} \mathrm{NMR}\left(126 \mathrm{MHz}, \mathrm{CDCl}_{3}\right) \delta 162.9(\mathrm{~d}, J=251.0 \mathrm{~Hz}), 154.1$, 136.6, 135.3, 133.8 (d, $J=8.5 \mathrm{~Hz}), 132.9,118.4,118.4,115.8$ (d, $J$ $=22.2 \mathrm{~Hz}$ ), 98.9, 94.4, 85.5, 31.6, 28.7. LC-MS (ESI): $\mathrm{m} / \mathrm{z}=281.2$ $[\mathrm{M}+\mathrm{H}]^{+}$. HRMS (ESI) $m / z:[\mathrm{M}+\mathrm{H}]^{+}$calculated for $\mathrm{C}_{16} \mathrm{H}_{13} \mathrm{FN}_{4}$, 281.1197; found 281.1201.
2-((4-Methoxyphenyl)ethynyl)- $N, 5$-dimethyl-5H-pyrrolo[2,3b]pyrazin-3-amine (5e). Mp: 148-150 ${ }^{\circ} \mathrm{C}$. ${ }^{1} \mathrm{H}$ NMR $(500 \mathrm{MHz}$, $\left.\mathrm{CDCl}_{3}\right) \delta 7.55(\mathrm{~d}, J=8.3 \mathrm{~Hz}, 2 \mathrm{H}), 7.03(\mathrm{~d}, J=3.0 \mathrm{~Hz}, 1 \mathrm{H}), 6.90(\mathrm{~d}$, $J=8.3 \mathrm{~Hz}, 2 \mathrm{H}), 6.47(\mathrm{~d}, J=2.9 \mathrm{~Hz}, 1 \mathrm{H}), 5.37(\mathrm{~d}, J=4.0 \mathrm{~Hz}, 1 \mathrm{H})$, 3.84 (s, 3H), 3.76 (s, 3H), 3.13 (d, $J=4.7 \mathrm{~Hz}, 3 \mathrm{H}) .{ }^{13} \mathrm{C}$ NMR (126 $\left.\mathrm{MHz}, \mathrm{CDCl}_{3}\right) \delta 160.0,152.2$, 139.4, 133.2, 130.1, 127.7, 120.1, 114.5, 114.1, 100.8, 94.4, 84.4, 55.3, 30.9, 28.2. LC-MS (ESI): $\mathrm{m} / \mathrm{z}$ $=293.2[\mathrm{M}+\mathrm{H}]^{+}$. HRMS (ESI) $\mathrm{m} / \mathrm{z}:[\mathrm{M}+\mathrm{H}]^{+}$calculated for $\mathrm{C}_{17} \mathrm{H}_{16} \mathrm{~N}_{4} \mathrm{O}$, 293.1397; found 293.1403.

$N, 5$-Dimethyl-2-(p-tolylethynyl)-5H-pyrrolo[2,3-b]pyrazin-3amine (5f). Mp: $132-134{ }^{\circ} \mathrm{C} .{ }^{1} \mathrm{H}$ NMR $\left(500 \mathrm{MHz}, \mathrm{CDCl}_{3}\right) \delta 7.51$ $(\mathrm{d}, J=6.8 \mathrm{~Hz}, 2 \mathrm{H}), 7.19$ (d, $J=7.0 \mathrm{~Hz}, 2 \mathrm{H}), 7.04(\mathrm{~s}, 1 \mathrm{H}), 6.48$ (s, $1 \mathrm{H}), 5.38(\mathrm{~s}, 1 \mathrm{H}), 3.77(\mathrm{~s}, 3 \mathrm{H}), 3.14(\mathrm{~s}, 3 \mathrm{H}), 2.40(\mathrm{~s}, 3 \mathrm{H}) .{ }^{13} \mathrm{C} \mathrm{NMR}$ $\left(126 \mathrm{MHz}, \mathrm{CDCl}_{3}\right) \delta 152.3,139.5,139.0,131.6,130.2,129.2$, 127.8, 119.9, 119.4, 100.9, 94.6, 85.1, 30.9, 28.2, 21.5. LC-MS (ESI): $m / z=277.2[\mathrm{M}+\mathrm{H}]^{+}$. HRMS (ESI) $m / z:[\mathrm{M}+\mathrm{H}]^{+}$calculated for $\mathrm{C}_{17} \mathrm{H}_{16} \mathrm{~N}_{4}, 277.1448$; found 277.1452.

2-(Cyclopropylethynyl)- $N, 5$-dimethyl-5H-pyrrolo[2,3-b]pyrazin3-amine (5g). Mp: $122-124{ }^{\circ} \mathrm{C} .{ }^{1} \mathrm{H}$ NMR $\left(500 \mathrm{MHz}, \mathrm{CDCl}_{3}\right) \delta 6.99$ $(\mathrm{d}, J=3.1 \mathrm{~Hz}, 1 \mathrm{H}), 6.42(\mathrm{~d}, J=3.1 \mathrm{~Hz}, 1 \mathrm{H}), 5.30(\mathrm{~s}, 1 \mathrm{H}), 3.74(\mathrm{~s}$, $3 \mathrm{H}), 3.09$ (d, $J=4.9 \mathrm{~Hz}, 3 \mathrm{H}), 1.62-1.43(\mathrm{~m}, 1 \mathrm{H}), 0.94(\mathrm{~d}, J=$ $8.4 \mathrm{~Hz}, 4 \mathrm{H}) .{ }^{13} \mathrm{C} \mathrm{NMR}\left(126 \mathrm{MHz}, \mathrm{CDCl}_{3}\right) \delta 152.4,139.1,129.6$, 127.3, 120.5, 100.7, 98.9, 72.2, 30.8, 28.2, 9.0, 0.3. LC-MS (ESI): $m / z=227.2[\mathrm{M}+\mathrm{H}]^{+}$. HRMS (ESI) $m / z:[\mathrm{M}+\mathrm{H}]^{+}$calculated for $\mathrm{C}_{13} \mathrm{H}_{14} \mathrm{~N}_{4}, 227.1291$; found 227.126.

2-((4-(Dimethylamino)phenyl)ethynyl)- $N$,5-dimethyl-5H-pyrrolo[2,3-b]pyrazin-3-amine (5h). Mp: $180-182{ }^{\circ} \mathrm{C}$. LC-MS (ESI): $m / z=306.3[\mathrm{M}+\mathrm{H}]^{+}$. HRMS (ESI) $m / z:[\mathrm{M}+\mathrm{H}]^{+}$calculated for $\mathrm{C}_{18} \mathrm{H}_{19} \mathrm{~N}_{5}, 306.1713$; found 306.1717.

N,5-Dimethyl-6-phenyl-2-(phenylethynyl)-5H-pyrrolo[2,3-b] pyrazin-3-amine (5i). Mp: $199-200{ }^{\circ} \mathrm{C}$. ${ }^{1} \mathrm{H}$ NMR $(500 \mathrm{MHz}$, $\left.\mathrm{CDCl}_{3}\right) \delta 7.64(\mathrm{~d}, J=3.4 \mathrm{~Hz}, 2 \mathrm{H}), 7.57(\mathrm{~d}, J=6.9 \mathrm{~Hz}, 2 \mathrm{H}), 7.50(\mathrm{t}, J$ $=6.9 \mathrm{~Hz}, 2 \mathrm{H}), 7.45-7.38(\mathrm{~m}, 4 \mathrm{H}), 6.58(\mathrm{~s}, 1 \mathrm{H}), 5.41(\mathrm{~s}, 1 \mathrm{H}), 3.81$ (s, 3H), $3.18(\mathrm{~s}, 3 \mathrm{H}) .{ }^{13} \mathrm{C}$ NMR $\left(126 \mathrm{MHz} \mathrm{CDCl}_{3}\right) \delta 152.4,141.4$, 141.1 , 132.2, 131.7, 130.6, 128.8, 128.7, 128.6, 128.4, 128.1, 122.5, 119.8, 100.7, 94.5, 85.8, 29.5, 28.3. LC-MS (ESI): $m / z=$ $339.2[\mathrm{M}+\mathrm{H}]^{+}$. HRMS (ESI) $m / z:[\mathrm{M}+\mathrm{H}]^{+}$calculated for $\mathrm{C}_{22} \mathrm{H}_{18} \mathrm{~N}_{4}, 339.1604$; found 339.1607.

2-((4-(Dimethylamino)phenyl)ethynyl)- $N, 5$-dimethyl-6-phenyl5H-pyrrolo[2,3-b]pyrazin-3-amine (5j). Mp: $129-131{ }^{\circ} \mathrm{C}$. LC-MS (ESI): $m / z=382.3[\mathbf{M}+\mathrm{H}]^{+}$. HRMS (ESI) $m / z:[\mathrm{M}+\mathrm{H}]^{+}$calculated for $\mathrm{C}_{24} \mathrm{H}_{23} \mathrm{~N}_{5}$, 382.2026; found 382.2026.

\subsection{General procedure for cyclization of DPP derivatives}

A solution of $N, 5$-dimethyl-2-((trimethylsilyl)ethynyl)-5 $H$-pyrrolo [2,3-b]pyrazin-3-amine $(0.09 \mathrm{~g}, 0.34 \mathrm{mmol})$ in NMP $(5 \mathrm{~mL})$ containing $t$-BuOK $(0.078 \mathrm{~g}, 0.7 \mathrm{mmol})$ was stirred at reflux for $2 \mathrm{~h}$. The mixture was quenched with water and extracted with EtOAc. The combined organic layers were concentrated in vacuum to give a residue that was subjected to silica gel chromatography eluting with $20 \%$ EtOAc to afford the titled compound as a pale yellow solid.

1,7-Dimethyl-1,7-dihydrodipyrrolo[2,3-b:3', $\left.2^{\prime}-e\right]$ pyrazine (6a). Mp: $168-170{ }^{\circ} \mathrm{C} .{ }^{1} \mathrm{H}$ NMR $\left(500 \mathrm{MHz}, \mathrm{CDCl}_{3}\right) \delta 7.35(\mathrm{~d}, J=3.5 \mathrm{~Hz}$, $1 \mathrm{H}), 6.69(\mathrm{~d}, J=3.6 \mathrm{~Hz}, 1 \mathrm{H}), 3.90(\mathrm{~s}, 3 \mathrm{H}) .{ }^{13} \mathrm{CNMR}(126 \mathrm{MHz}$, $\left.\mathrm{CDCl}_{3}\right) \delta 138.4,136.2,130.8,99.7,31.3$. LC-MS (ESI): $m / z=187.1$ 
$[\mathrm{M}+\mathrm{H}]^{+}$. HRMS (ESI) $m / z:[\mathrm{M}+\mathrm{H}]^{+}$calculated for $\mathrm{C}_{10} \mathrm{H}_{10} \mathrm{~N}_{4}$, 187.0978; found 187.0988.

1,7-Dimethyl-2-phenyl-1,7-dihydrodipyrrolo[2,3-b:3', $\left.2^{\prime}-e\right]$ pyrazine (6b). Mp: $165-166{ }^{\circ} \mathrm{C} .{ }^{1} \mathrm{H}$ NMR $\left(500 \mathrm{MHz}, \mathrm{CDCl}_{3}\right) \delta 7.61$ (d, $J=7.3 \mathrm{~Hz}, 2 \mathrm{H}), 7.51(\mathrm{t}, J=7.5 \mathrm{~Hz}, 2 \mathrm{H}), 7.44(\mathrm{~d}, J=7.4 \mathrm{~Hz}, 1 \mathrm{H})$, $7.35(\mathrm{~d}, J=3.6 \mathrm{~Hz}, 1 \mathrm{H}), 6.77(\mathrm{~s}, 1 \mathrm{H}), 6.69(\mathrm{~d}, J=3.6 \mathrm{~Hz}, 1 \mathrm{H}), 3.92$ (s, 3H), $3.91(\mathrm{~s}, 3 \mathrm{H}) .{ }^{13} \mathrm{C}$ NMR (126 MHz, $\left.\mathrm{CDCl}_{3}\right) \delta 143.8,140.3$, 138.3, 136.6, 136.4, 132.3, 130.6, 129.1, 128.6, 128.4, 99.9, 99.8, 31.4, 29.8. LC-MS (ESI): $m / z=263.2[\mathrm{M}+\mathrm{H}]^{+}$. HRMS (ESI) $m / z$ : $[\mathrm{M}+\mathrm{H}]^{+}$calculated for $\mathrm{C}_{16} \mathrm{H}_{14} \mathrm{~N}_{4}, 263.1291$; found 263.1303.

2-(3-Fluorophenyl)-1,7-dimethyl-1,7-dihydrodipyrrolo[2,3$\boldsymbol{b}: \mathbf{3}^{\prime}, \mathbf{2}^{\prime}$-e $]$ pyrazine (6c). Mp: $187-188{ }^{\circ} \mathrm{C} .{ }^{1} \mathrm{H}$ NMR $(500 \mathrm{MHz}$, $\left.\mathrm{CDCl}_{3}\right) \delta 7.47(\mathrm{dd}, J=14.2,7.7 \mathrm{~Hz}, 1 \mathrm{H}), 7.43-7.36(\mathrm{~m}, 2 \mathrm{H}), 7.32$ $(\mathrm{d}, J=8.7 \mathrm{~Hz}, 1 \mathrm{H}), 7.14(\mathrm{t}, J=7.2 \mathrm{~Hz}, 1 \mathrm{H}), 6.81(\mathrm{~d}, J=4.6 \mathrm{~Hz}$, $1 \mathrm{H}), 6.71(\mathrm{t}, J=5.0 \mathrm{~Hz}, 1 \mathrm{H}), 3.93(\mathrm{dd}, J=9.7,4.5 \mathrm{~Hz}, 6 \mathrm{H}) .{ }^{13} \mathrm{C}$ NMR $\left(126 \mathrm{MHz}, \mathrm{CDCl}_{3}\right) \delta 162.7(\mathrm{~d}, J=246.8 \mathrm{~Hz}), 142.2,140.3$, $138.5,136.9,136.0,134.3(\mathrm{~d}, J=8.1 \mathrm{~Hz}), 131.0,130.2(\mathrm{~d}, J=8.5$ $\mathrm{Hz}), 124.7,115.9(\mathrm{~d}, J=22.4 \mathrm{~Hz}), 115.2(\mathrm{~d}, J=21.0 \mathrm{~Hz}), 100.5$, 99.8, 31.3, 29.9. LC-MS (ESI): $m / z=281.1[\mathrm{M}+\mathrm{H}]^{+}$. HRMS (ESI) $m / z:[\mathrm{M}+\mathrm{H}]^{+}$calculated for $\mathrm{C}_{16} \mathrm{H}_{13} \mathrm{FN}_{4}, 281.1197$; found 281.1206.

2-(4-Fluorophenyl)-1,7-dimethyl-1,7-dihydrodipyrrolo[2,3$\boldsymbol{b}: 3^{\prime}, \mathbf{2}^{\prime}-\boldsymbol{e}$ ]pyrazine (6d). Mp: $158-159{ }^{\circ} \mathrm{C} .{ }^{1} \mathrm{H}$ NMR $(500 \mathrm{MHz}$, $\left.\mathrm{CDCl}_{3}\right) \delta 7.60-7.51(\mathrm{~m}, 2 \mathrm{H}), 7.35(\mathrm{~d}, J=3.6 \mathrm{~Hz}, 1 \mathrm{H}), 7.23-7.12$ $(\mathrm{m}, 2 \mathrm{H}), 6.73(\mathrm{~s}, 1 \mathrm{H}), 6.69(\mathrm{~d}, J=3.7 \mathrm{~Hz}, 1 \mathrm{H}), 3.92(\mathrm{~s}, 3 \mathrm{H}), 3.87(\mathrm{~s}$, $3 \mathrm{H}) .{ }^{13} \mathrm{C}$ NMR $\left(126 \mathrm{MHz}, \mathrm{CDCl}_{3}\right) \delta 163.8,161.8,142.6,140.1$, 138.3, 136.7, 136.3, 130.8 (d, $J=8.2 \mathrm{~Hz}), 130.7,128.4$ (d, $J=3.3$ $\mathrm{Hz}), 115.7(\mathrm{~d}, J=21.7 \mathrm{~Hz}), 99.8(\mathrm{~d}, J=15.8 \mathrm{~Hz}), 31.3,29.7$. LCMS (ESI): $m / z=281.1[\mathrm{M}+\mathrm{H}]^{+}$. HRMS (ESI) $m / z:[\mathrm{M}+\mathrm{H}]^{+}$ calculated for $\mathrm{C}_{16} \mathrm{H}_{13} \mathrm{FN}_{4}$, 281.1197; found 281.1209.

2-(4-Methoxyphenyl)-1,7-dimethyl-1,7-dihydrodipyrrolo[2,3$\boldsymbol{b}: 3^{\prime}, \mathbf{2}^{\prime}$-e]pyrazine (6e). Mp: $155-156{ }^{\circ} \mathrm{C} .{ }^{1} \mathrm{H}$ NMR $(500 \mathrm{MHz}$, $\left.\mathrm{CDCl}_{3}\right) \delta 7.50(\mathrm{~d}, J=8.7 \mathrm{~Hz}, 2 \mathrm{H}), 7.29(\mathrm{~d}, J=3.6 \mathrm{~Hz}, 1 \mathrm{H}), 7.00(\mathrm{~d}$, $J=8.7 \mathrm{~Hz}, 2 \mathrm{H}), 6.70(\mathrm{~s}, 1 \mathrm{H}), 6.67(\mathrm{~d}, J=3.6 \mathrm{~Hz}, 1 \mathrm{H}), 3.87(\mathrm{~s}, 3 \mathrm{H})$, $3.85(\mathrm{~s}, 3 \mathrm{H}), 3.84(\mathrm{~s}, 3 \mathrm{H}) .{ }^{13} \mathrm{C}$ NMR $\left(126 \mathrm{MHz}, \mathrm{CDCl}_{3}\right) \delta 159.8$, 143.8, 140.1, 138.1, 136.5, 136.4, 130.4, 130.3, 124.6, 114.1, 99.6, 99.0, 55.3, 31.3, 29.7. LC-MS (ESI): $m / z=293.2[\mathrm{M}+\mathrm{H}]^{+}$. HRMS (ESI) $m / z:[\mathrm{M}+\mathrm{H}]^{+}$calculated for $\mathrm{C}_{17} \mathrm{H}_{16} \mathrm{~N}_{4} \mathrm{O}$, 293.1397; found 293.1406.

1,7-Dimethyl-2-p-tolyl-1,7-dihydrodipyrrolo[2,3-b:3', $\left.2^{\prime}-e\right]$ pyrazine (6f). Mp: $174-176{ }^{\circ} \mathrm{C} .{ }^{1} \mathrm{H}$ NMR $\left(500 \mathrm{MHz}, \mathrm{CDCl}_{3}\right) \delta 7.52(\mathrm{~d}$, $J=8.0 \mathrm{~Hz}, 2 \mathrm{H}), 7.36(\mathrm{~d}, J=3.6 \mathrm{~Hz}, 1 \mathrm{H}), 7.33(\mathrm{~d}, J=7.9 \mathrm{~Hz}, 2 \mathrm{H})$, $6.76(\mathrm{~s}, 1 \mathrm{H}), 6.71(\mathrm{~d}, J=3.6 \mathrm{~Hz}, 1 \mathrm{H}), 3.94(\mathrm{~s}, 3 \mathrm{H}), 3.92(\mathrm{~s}, 3 \mathrm{H})$, $2.46(\mathrm{~s}, 3 \mathrm{H}) .{ }^{13} \mathrm{C}$ NMR $\left(126 \mathrm{MHz}, \mathrm{CDCl}_{3}\right) \delta 144.0,140.2,138.4$, 138.2, 136.6, 136.5, 130.51, 129.4, 129.4, 129.0, 99.7, 99.5, 31.3, 29.8, 21.3. LC-MS (ESI): $m / z=277.3[\mathrm{M}+\mathrm{H}]^{+}$. HRMS (ESI) $m / z$ : $[\mathrm{M}+\mathrm{H}]^{+}$calculated for $\mathrm{C}_{17} \mathrm{H}_{16} \mathrm{~N}_{4}, 277.1448$; found 277.1455.

2-Cyclopropyl-1,7-dimethyl-1,7-dihydrodipyrrolo[2,3-b:3', $2^{\prime}$ e]pyrazine (6g). Mp: $163-165{ }^{\circ} \mathrm{C} .{ }^{1} \mathrm{H}$ NMR $\left(500 \mathrm{MHz}, \mathrm{CDCl}_{3}\right)$ $\delta 6.65(\mathrm{~d}, J=3.1 \mathrm{~Hz}, 1 \mathrm{H}), 6.26(\mathrm{~s}, 1 \mathrm{H}), 3.93(\mathrm{~d}, J=4.5 \mathrm{~Hz}, 3 \mathrm{H})$, $3.91(\mathrm{~d}, J=4.5 \mathrm{~Hz}, 3 \mathrm{H}), 1.99$ (d, $J=5.1 \mathrm{~Hz}, 1 \mathrm{H}), 1.07$ (d, $J=$ $6.5 \mathrm{~Hz}, 2 \mathrm{H}), 0.85(\mathrm{~d}, J=3.2 \mathrm{~Hz}, 2 \mathrm{H}) .{ }^{13} \mathrm{C}$ NMR $\left(126 \mathrm{MHz}, \mathrm{CDCl}_{3}\right)$ $\delta$ 146.5, 139.3, 137.8, 136.5, 135.7, 129.6, 99.6, 95.2, 31.3, 28.0, 8.1, 6.9. LC-MS (ESI): $m / z=227.2[\mathrm{M}+\mathrm{H}]^{+}$. HRMS (ESI) $m / z:[\mathrm{M}+$ $\mathrm{H}]^{+}$calculated for $\mathrm{C}_{13} \mathrm{H}_{14} \mathrm{~N}_{4}, 227.1291$; found 227.1304.

4-(1,7-Dimethyl-1,7-dihydrodipyrrolo[2,3-b:3', $\left.2^{\prime}-e\right]$ pyrazin-2yl)- $N, N$-dimethylaniline (6h). Mp: $\quad 188-190 \quad{ }^{\circ} \mathrm{C} . \quad{ }^{1} \mathrm{H} \quad \mathrm{NMR}$ $\left(500 \mathrm{MHz}, \mathrm{CDCl}_{3}\right) \delta 7.50(\mathrm{~d}, J=8.8 \mathrm{~Hz}, 2 \mathrm{H}), 7.36(\mathrm{~d}, J=3.6 \mathrm{~Hz}$, $1 \mathrm{H}), 6.83(\mathrm{~d}, J=8.8 \mathrm{~Hz}, 2 \mathrm{H}), 6.66(\mathrm{~d}, J=3.6 \mathrm{~Hz}, 1 \mathrm{H}), 6.65(\mathrm{~s}, 1 \mathrm{H})$, $3.94(\mathrm{~d}, J=3.7 \mathrm{~Hz}, 6 \mathrm{H}), 3.04(\mathrm{~s}, 6 \mathrm{H}) .{ }^{13} \mathrm{C} \mathrm{NMR}\left(126 \mathrm{MHz}, \mathrm{CDCl}_{3}\right)$ $\delta$ 150.4, 146.1, 141.5, 139.8, 135.7, 133.8, 130.82, 130.0, 119.7, 112.0, 98.9, 97.5, 40.3, 31.7, 30.0. LC-MS (ESI): $\mathrm{m} / z=306.2[\mathrm{M}+$ $\mathrm{H}]^{+}$. HRMS (ESI) $m / z:[\mathrm{M}+\mathrm{H}]^{+}$calculated for $\mathrm{C}_{18} \mathrm{H}_{19} \mathrm{~N}_{5}$, 306.1713; found 306.1717.

1,7-Dimethyl-2,6-diphenyl-1,7-dihydrodipyrrolo[2,3-b:3', $\left.\mathbf{2}^{\prime}-e\right]$ pyrazine (6i). Mp: $197-199^{\circ} \mathrm{C} .{ }^{1} \mathrm{H}$ NMR $\left(500 \mathrm{MHz}, \mathrm{CDCl}_{3}\right) \delta 7.66$ $(\mathrm{d}, J=7.2 \mathrm{~Hz}, 2 \mathrm{H}), 7.55(\mathrm{t}, J=7.5 \mathrm{~Hz}, 2 \mathrm{H}), 7.48(\mathrm{~d}, J=7.4 \mathrm{~Hz}$, $1 \mathrm{H}), 6.81(\mathrm{~s}, 1 \mathrm{H}), 3.97(\mathrm{~s}, 3 \mathrm{H}) .{ }^{13} \mathrm{C}$ NMR $\left(126 \mathrm{MHz}, \mathrm{CDCl}_{3}\right)$ $\delta$ 144.7, 141.8, 134.9, 132.2, 129.1, 128.7, 128.5, 99.3, 30.1. LCMS (ESI): $m / z=339.2[\mathrm{M}+\mathrm{H}]^{+}$. HRMS (ESI) $m / z:[\mathrm{M}+\mathrm{H}]^{+}$ calculated for $\mathrm{C}_{22} \mathrm{H}_{18} \mathrm{~N}_{4}, 339.1604$; found 339.1607.

4-(1,7-Dimethyl-6-phenyl-1,7-dihydrodipyrrolo[2,3-b:3', $\left.2^{\prime}-e\right]$ pyrazin-2-yl)- $N, N$-dimethylaniline (6j). Mp: $231-233{ }^{\circ} \mathrm{C} .{ }^{1} \mathrm{H}$ NMR $\left(500 \mathrm{MHz}, \mathrm{CDCl}_{3}\right) \delta 7.62(\mathrm{~s}, 2 \mathrm{H}), 7.51(\mathrm{~s}, 4 \mathrm{H}), 7.44(\mathrm{~d}, J=6.2 \mathrm{~Hz}$, $1 \mathrm{H}), 6.84(\mathrm{~d}, J=6.9 \mathrm{~Hz}, 2 \mathrm{H}), 6.77(\mathrm{~s}, 1 \mathrm{H}), 6.67(\mathrm{~s}, 1 \mathrm{H}), 3.93(\mathrm{~s}$, $6 \mathrm{H}), 3.05(\mathrm{~s}, 6 \mathrm{H}) .{ }^{13} \mathrm{C}$ NMR $\left(126 \mathrm{MHz}, \mathrm{CDCl}_{3}\right) \delta 150.3,144.8$, $142.9,140.2,139.8,137.4,136.3,132.5,129.9,129.0,128.6$, 128.2, 119.9, 112.1, 99.9, 98.2, 40.3, 29.9. LC-MS (ESI): $\mathrm{m} / \mathrm{z}=$ $382.2[\mathrm{M}+\mathrm{H}]^{+}$. HRMS (ESI) $\mathrm{m} / \mathrm{z}:[\mathrm{M}+\mathrm{H}]^{+}$calculated for $\mathrm{C}_{24} \mathrm{H}_{23} \mathrm{~N}_{5}, 382.2026$; found 382.2037.

\subsection{General method for Buchwald reaction}

tert-Butyl 3-chloro-5-methyl-5H-pyrrolo[2,3-b]pyrazin-2-ylcarbamate (7a). An air-dried RB flask was charged sequentially with 1,4-dioxane (50 mL), $t$-amyl alcohol $(10 \mathrm{~mL}), 2$-bromo-3chloro-5-methyl-5 $H$-pyrrolo[2,3- $b$ ]pyrazine $(2.2 \mathrm{~g}$, $8.9 \mathrm{mmol}$ ), $\mathrm{Pd}(\mathrm{OAc})_{2}(0.2 \mathrm{~g}, 0.89 \mathrm{mmol})$, xantphos $(0.51 \mathrm{~g}, 0.89 \mathrm{mmol})$, $\mathrm{Cs}_{2} \mathrm{CO}_{3}(5.82 \mathrm{~g}, 17 \mathrm{mmol})$ and $t$-butyl carbamate $(1.04 \mathrm{~g}, 8.9$ mmol). The resultant suspension was stirred at reflux at $90{ }^{\circ} \mathrm{C}$ for $3 \mathrm{~h}$. Once the reaction was determined to be complete by using TLC, the mixture was cooled to room temperature, diluted with EtOAc and filtered through a pad of Celite. The filtrate was concentrated in vacuum. The residue was subjected to silica gel column chromatography to afford desired product as a crystalline solid. Yield: $78 \%,{ }^{1} \mathrm{H}$ NMR $\left(500 \mathrm{MHz}, \mathrm{CDCl}_{3}\right) \delta 7.41(\mathrm{~s}, 1 \mathrm{H})$, $7.13(\mathrm{~s}, 1 \mathrm{H}), 6.64(\mathrm{~s}, 1 \mathrm{H}), 3.87(\mathrm{~s}, 3 \mathrm{H}), 1.50(\mathrm{~s}, 9 \mathrm{H}) .{ }^{13} \mathrm{C} \mathrm{NMR}$ $\left(126 \mathrm{MHz}, \mathrm{CDCl}_{3}\right) \delta 151.4,138.7,136.9,136.0,133.6,132.2$, 100.8, 81.5, 31.7, 28.2. LC-MS (ESI): $m / z=305.2[\mathrm{M}+\mathrm{Na}]^{+}$. HRMS (ESI) $m / z:[\mathrm{M}+\mathrm{H}]^{+}$calculated for $\mathrm{C}_{12} \mathrm{H}_{15} \mathrm{ClN}_{4} \mathrm{O}_{2}$, 283.0956; found 283.0961.

tert-Butyl3-chloro-5-methyl-6-phenyl-5H-pyrrolo[2,3-b]pyrazin2-ylcarbamate (7b). Yield: $76 \%,{ }^{1} \mathrm{H}$ NMR $\left(500 \mathrm{MHz}, \mathrm{CDCl}_{3}\right)$ $\delta 7.53(\mathrm{~m}, J=15.4,7.1 \mathrm{~Hz}, 5 \mathrm{H}), 7.22(\mathrm{~s}, 1 \mathrm{H}), 6.74(\mathrm{~s}, 1 \mathrm{H}), 3.83(\mathrm{~s}$, $3 \mathrm{H}), 1.57$ (s, 9H). ${ }^{13} \mathrm{C}$ NMR (126 MHz, $\left.\mathrm{CDCl}_{3}\right) \delta$ 151.5, 146.6, 139.0, 138.5, 136.2, 131.6, 131.1, 129.1, 129.1, 128.8, 100.6, 81.4, 30.1, 28.3. LC-MS (ESI): $m / z=381.2[\mathrm{M}+\mathrm{Na}]^{+}$. HRMS (ESI) $m / z$ : $[\mathrm{M}+\mathrm{H}]^{+}$calculated for $\mathrm{C}_{18} \mathrm{H}_{19} \mathrm{ClN}_{4} \mathrm{O}_{2}, 359.1269$; found 359.1290 .

tert-Butyl3-chloro-5-methyl-5H-pyrrolo[2,3-b]pyrazin-2-yl(methyl) carbamate (8a). To a solution of 2-bromo-3-chloro-5Hpyrrolo[2,3-b]pyrazine $(2.76 \mathrm{~g}, 9.7 \mathrm{mmol})$ in DMF $(35 \mathrm{~mL})$ under nitrogen atmosphere at $0{ }^{\circ} \mathrm{C}$ was added $\mathrm{NaH}(60 \%$ dispersion in mineral oil, $0.44 \mathrm{~g}, 10 \mathrm{mmol}$ ) carefully. After $30 \mathrm{~min}$, MeI 
(0.6 mL, $9.7 \mathrm{mmol}$ ) was added and the mixture is warmed slowly to ambient temperature. After $3 \mathrm{~h}$, the mixture was poured into ice-cold water and the precipitate is collected by vacuum filtration. The crude solid was subjected to silica gel chromatography. Yield: $92 \%,{ }^{1} \mathrm{H}$ NMR $\left(500 \mathrm{MHz}, \mathrm{CDCl}_{3}\right) \delta 7.49(\mathrm{~s}, 1 \mathrm{H})$, $6.65(\mathrm{~s}, 1 \mathrm{H}), 3.90(\mathrm{~s}, 3 \mathrm{H}), 3.29(\mathrm{~d}, J=0.8 \mathrm{~Hz}, 3 \mathrm{H}), 1.37(\mathrm{~s}, 9 \mathrm{H}) .{ }^{13} \mathrm{C}$ NMR (126 MHz, $\left.\mathrm{CDCl}_{3}\right) \delta$ 154.1, 143.1, 139.6, 138.5, 136.5, 134.2, 100.8, 80.9, 35.1, 31.8, 28.1. HRMS (ESI) $m / z:[\mathrm{M}+\mathrm{H}]^{+}$calculated for $\mathrm{C}_{13} \mathrm{H}_{17} \mathrm{ClN}_{4} \mathrm{O}_{2}, 297.1113$; found 297.1118.

tert-Butyl 3-chloro-5-methyl-6-phenyl-5 $\mathrm{H}$-pyrrolo[2,3-b] pyrazin-2-yl(methyl)carbamate (8b). Yield: $95 \%,{ }^{1} \mathrm{H}$ NMR $(500$ $\left.\mathrm{MHz} \mathrm{CDCl}_{3}\right) \delta 7.56(\mathrm{dt}, J=10.7,7.0 \mathrm{~Hz}, 5 \mathrm{H}), 6.73(\mathrm{~s}, 1 \mathrm{H}), 3.89(\mathrm{~s}$, $3 \mathrm{H}), 3.32(\mathrm{~s}, 3 \mathrm{H}), 1.41(\mathrm{~s}, 9 \mathrm{H}) .{ }^{13} \mathrm{C} \mathrm{NMR}\left(126 \mathrm{MHz}, \mathrm{CDCl}_{3}\right)$ $\delta$ 154.1, 147.3, 143.5, 140.1, 139.0, 136.7, 130.9, 129.4, 129.1, 128.9, 100.4, 80.9, 30.9, 30.2, 28.2. LC-MS (ESI): $m / z=317.1[\mathrm{M}$ - butyl $]^{+}$. HRMS (ESI) $m / z:[\mathrm{M}+\mathrm{H}]^{+}$calculated for $\mathrm{C}_{19} \mathrm{H}_{21} \mathrm{ClN}_{4} \mathrm{O}_{2}$, 373.1426; found 373.1445.

3-Chloro- $N, 5$-dimethyl-5 $H$-pyrrolo[2,3-b]pyrazin-2-amine (9a). A solution of tert-butyl 3-chloro-5-methyl-5H-pyrrolo[2,3- $b]$ pyrazin-2-yl(methyl)carbamate $(2.6 \mathrm{~g}, 8.7 \mathrm{mmol})$ in DCM (50 $\mathrm{mL})$ containing TFA $(5.0 \mathrm{~mL}, 43 \mathrm{mmol})$ was stirred at room temperature for $10 \mathrm{~min}$ then refluxed for $50 \mathrm{~min}$. The mixture was made basic by addition of $1 \mathrm{M} \mathrm{NaOH}$ solution and extracted with DCM. The organic layer was dried over $\mathrm{MgSO}_{4}$, and concentrated in vacuum to get the desired product. Yield: $96 \%$, ${ }^{1} \mathrm{H}$ NMR $\left(500 \mathrm{MHz}, \mathrm{CDCl}_{3}\right) \delta 7.20(\mathrm{~d}, J=3.3 \mathrm{~Hz}, 1 \mathrm{H}), 6.44(\mathrm{~d}, J=$ $5.0 \mathrm{~Hz}, 1 \mathrm{H}), 4.98(\mathrm{~s}, 1 \mathrm{H}), 3.80(\mathrm{~d}, J=1.8 \mathrm{~Hz}, 3 \mathrm{H}), 3.11(\mathrm{~s}, 3 \mathrm{H}) .{ }^{13} \mathrm{C}$ NMR $\left(126 \mathrm{MHz}, \mathrm{CDCl}_{3}\right) \delta 147.9,135.2,133.5,130.7,128.0$, 98.7, 31.6, 29.0. LC-MS (ESI): $m / z=197.0[\mathrm{M}+\mathrm{H}]^{+}$. HRMS (ESI) $m / z:[\mathrm{M}$ $+\mathrm{H}]^{+}$calculated for $\mathrm{C}_{8} \mathrm{H}_{9} \mathrm{ClN}_{4}, 197.0589$; found 197.0595.

3-Chloro- $\mathrm{N}, 5$-dimethyl-5H-pyrrolo[2,3-b]pyrazin-2-amine (9b). Yield: $98 \%,{ }^{1} \mathrm{H}$ NMR (500 MHz, $\left.\mathrm{CDCl}_{3}\right) \delta 7.55(\mathrm{~d}, J=7.2 \mathrm{~Hz}$, $2 \mathrm{H}), 7.51(\mathrm{t}, J=7.4 \mathrm{~Hz}, 2 \mathrm{H}), 7.45(\mathrm{t}, J=7.2 \mathrm{~Hz}, 1 \mathrm{H}), 6.55(\mathrm{~s}, 1 \mathrm{H})$, $5.00(\mathrm{~s}, 1 \mathrm{H}), 3.81(\mathrm{~s}, 3 \mathrm{H}), 3.13(\mathrm{~d}, J=4.7 \mathrm{~Hz}, 3 \mathrm{H}) .{ }^{13} \mathrm{C} \mathrm{NMR}(126$ $\left.\mathrm{MHz}, \mathrm{CDCl}_{3}\right) \delta 148.0,143.6,135.5,135.2,131.9,129,128.7$, 128.5, 128.0, 99.0, 30.0, 28.9; LC-MS (ESI): $m / z=273.2[\mathrm{M}+$ $\mathrm{H}]^{+}$. HRMS (ESI) $m / z$ : $[\mathrm{M}+\mathrm{H}]^{+}$calculated for $\mathrm{C}_{14} \mathrm{H}_{13} \mathrm{ClN}_{4}$, 273.0902 ; found 273.0919 .

\subsection{General procedure for Sonogashira reaction}

To a stirred solution of 3-chloro- $N, 5$-dimethyl-5 $H$-pyrrolo[2,3- $b$ ] pyrazin-2-amine $(0.2 \mathrm{~g}, 1.0 \mathrm{mmol})$ in DMF $(2 \mathrm{~mL})$ was added TEA (0.3 mL, $3.0 \mathrm{mmol})$, CuI (0.019 g, $0.1 \mathrm{mmol}), \mathrm{Pd}\left(\mathrm{PPh}_{3}\right)_{2} \mathrm{Cl}_{2}$ (0.07 g, $0.1 \mathrm{mmol})$ and TMS-acetylene $(0.1 \mathrm{~mL}, 1.0 \mathrm{mmol})$. The mixture subjected to microwave irradiation for $50 \mathrm{~min}$ at $90{ }^{\circ} \mathrm{C}$. Then the reaction mixture was cooled to ambient temperature and diluted with water and extracted with EtOAc twice. The combined organic layer was concentrated in vacuum to give a residue that was subjected to silica column chromatography to give desired compound as a brown solid.

3-((4-Fluorophenyl)ethynyl)- $N, 5$-dimethyl-5 $H$-pyrrolo[2,3- $b]$ pyrazin-2-amine (10c). ${ }^{1} \mathrm{H} \mathrm{NMR}\left(500 \mathrm{MHz}, \mathrm{CDCl}_{3}\right) \delta 7.47$ (dd, $J=$ 14.2, 7.7 Hz, 1H), 7.43-7.36 (m, 2H), 7.32 (d, $J=8.7 \mathrm{~Hz}, 1 \mathrm{H}), 7.14$ $(\mathrm{t}, J=7.2 \mathrm{~Hz}, 1 \mathrm{H}), 6.81(\mathrm{~d}, J=4.6 \mathrm{~Hz}, 1 \mathrm{H}), 6.71(\mathrm{t}, J=5.0 \mathrm{~Hz}$, $1 \mathrm{H}), 3.93(\mathrm{dd}, J=9.7,4.5 \mathrm{~Hz}, 6 \mathrm{H}) .{ }^{13} \mathrm{C} \mathrm{NMR}\left(126 \mathrm{MHz}, \mathrm{CDCl}_{3}\right.$ ) $\delta 162.9(\mathrm{~d}, J=251.0 \mathrm{~Hz}), 152.8,136.6,135.6,133.8$ (d, $J=8.5$
Hz), 132.9, 119.0, 118.1, 115.8 (d, $J=22.2 \mathrm{~Hz}$ ), 98.9, 95.3, 86.3, 33.1, 29.88. LC-MS (ESI): $m / z=281.1[\mathrm{M}+\mathrm{H}]^{+}$. HRMS (ESI) $m / z$ : $[\mathrm{M}+\mathrm{H}]^{+}$calculated for $\mathrm{C}_{16} \mathrm{H}_{13} \mathrm{FN}_{4}, 281.1197$; found 281.1201.

$N, 5$-Dimethyl-6-phenyl-3-(phenylethynyl)-5 $\mathrm{H}$-pyrrolo[2,3- $b]$ pyrazin-2-amine (10f). Mp: $171-173{ }^{\circ} \mathrm{C},{ }^{1} \mathrm{H}$ NMR $(500 \mathrm{MHz}$, $\left.\mathrm{CDCl}_{3}\right) \delta 7.66-7.63(\mathrm{~m}, 2 \mathrm{H}), 7.59-7.56(\mathrm{~m}, 2 \mathrm{H}), 7.52(\mathrm{~d}, J=$ $7.2 \mathrm{~Hz}, 2 \mathrm{H}), 7.47-7.44(\mathrm{~m}, 1 \mathrm{H}), 7.40(\mathrm{dd}, J=4.2,2.3 \mathrm{~Hz}, 3 \mathrm{H}), 6.56$ $(\mathrm{s}, 1 \mathrm{H}), 5.31(\mathrm{~d}, J=5.5 \mathrm{~Hz}, 1 \mathrm{H}), 3.85(\mathrm{~s}, 3 \mathrm{H}), 3.17$ (d, $J=5.0 \mathrm{~Hz}$, $3 \mathrm{H}) .{ }^{13} \mathrm{C}$ NMR $\left(126 \mathrm{MHz}, \mathrm{CDCl}_{3}\right.$ ) $\delta$ 153.2, 145.7, 137.1, 136.8, 131.9, 131.8, 129.0, 128.9, 128.7, 128.6, 128.4, 122.3, 117.8, 99.4, 95.7, 86.1, 30.0, 29.7, 28.8; LC-MS (ESI): $m / z=339.3[\mathrm{M}+\mathrm{H}]^{+}$. HRMS (ESI) $m / z$ : $[\mathrm{M}+\mathrm{H}]^{+}$calculated for $\mathrm{C}_{22} \mathrm{H}_{18} \mathrm{~N}_{4}, 339.1604$; found 339.1624 .

3-((4-Methoxyphenyl)ethynyl)- $N, 5$-dimethyl-6-phenyl-5H-pyrrolo[2,3-b]pyrazin-2-amine (10g). LC-MS (ESI): $m / z=369.2[\mathrm{M}+$ $\mathrm{H}]^{+}$. HRMS (ESI) $m / z$ : $[\mathrm{M}+\mathrm{H}]^{+}$calculated for $\mathrm{C}_{23} \mathrm{H}_{20} \mathrm{~N}_{4} \mathrm{O}$, 369.1710; found 369.1715 .

$N, 5$-Dimethyl-3-( $p$-tolylethynyl)-5H-pyrrolo[2,3-b]pyrazin-2amine (10h). Mp: $144-146{ }^{\circ} \mathrm{C},{ }^{1} \mathrm{H}$ NMR $\left(500 \mathrm{MHz}, \mathrm{CDCl}_{3}\right) \delta 7.58$ $(\mathrm{d}, J=7.2 \mathrm{~Hz}, 2 \mathrm{H}), 7.56-7.50(\mathrm{~m}, 4 \mathrm{H}), 7.46(\mathrm{t}, J=7.3 \mathrm{~Hz}, 1 \mathrm{H})$, $7.22(\mathrm{~d}, J=7.9 \mathrm{~Hz}, 2 \mathrm{H}), 6.56(\mathrm{~s}, 1 \mathrm{H}), 5.31$ (d, $J=4.8 \mathrm{~Hz}, 1 \mathrm{H}), 3.85$ $(\mathrm{s}, 3 \mathrm{H}), 3.17(\mathrm{~d}, J=5.0 \mathrm{~Hz}, 3 \mathrm{H}), 2.41(\mathrm{~s}, 3 \mathrm{H}) .{ }^{13} \mathrm{C} \mathrm{NMR}(126 \mathrm{MHz}$, $\left.\mathrm{CDCl}_{3}\right) \delta 153.2,145.5,139.2,137.0,136.6,131.9,131.7,129.2$, 129.0, 128.7, 128.6, 119.2, 118.0, 99.4, 96.1, 85.4, 30.0, 28.8, 21.6. LC-MS (ESI): $m / z=353.2[\mathrm{M}+\mathrm{H}]^{+}$. HRMS (ESI) $m / z:[\mathrm{M}+\mathrm{H}]^{+}$ calculated for $\mathrm{C}_{23} \mathrm{H}_{20} \mathrm{~N}_{4}, 353.1761$; found 353.1766.

3-((4-(Dimethylamino)phenyl)ethynyl)- $N$,5-dimethyl-6-phenyl$5 \boldsymbol{H}$-pyrrolo[2,3-b]pyrazin-2-amine (10i). LC-MS (ESI): $\mathrm{m} / \mathrm{z}=$ $382.3[\mathrm{M}+\mathrm{H}]^{+}$.

3-((3-Fluorophenyl)ethynyl)- $N, 5$-dimethyl-6-phenyl-5H-pyrrolo[2,3-b]pyrazin-2-amine (10j). Mp: $158-160{ }^{\circ} \mathrm{C},{ }^{1} \mathrm{H}$ NMR $(500$ $\left.\mathrm{MHz} \mathrm{CDCl}_{3}\right) \delta 7.58(\mathrm{~d}, J=7.2 \mathrm{~Hz}, 2 \mathrm{H}), 7.53(\mathrm{t}, J=7.5 \mathrm{~Hz}, 2 \mathrm{H})$, $7.47(\mathrm{t}, J=7.3 \mathrm{~Hz}, 1 \mathrm{H}), 7.43(\mathrm{~d}, J=7.7 \mathrm{~Hz}, 1 \mathrm{H}), 7.40-7.33(\mathrm{~m}$, 2H), 7.14-7.08 (m, 1H), $6.56(\mathrm{~s}, 1 \mathrm{H}), 5.25(\mathrm{~d}, J=4.7 \mathrm{~Hz}, 1 \mathrm{H}), 3.85$ $(\mathrm{s}, 3 \mathrm{H}), 3.17$ (d, $J=5.0 \mathrm{~Hz}, 3 \mathrm{H}) .{ }^{13} \mathrm{C} \mathrm{NMR}\left(126 \mathrm{MHz}, \mathrm{CDCl}_{3}\right)$ $\delta 162.3(\mathrm{~d}, J=247.0 \mathrm{~Hz}), 153.3,146.1,137.1(\mathrm{~d}, J=4.0 \mathrm{~Hz})$, 131.8, $130.0(\mathrm{~d}, J=8.6 \mathrm{~Hz}), 129.2,129.0,128.7,127.7$ (d, $J=3.1$ $\mathrm{Hz}), 124.2,124.1,118.54(\mathrm{~d}, J=23.0 \mathrm{~Hz}), 117.1,116.26$ (d, $J=$ $21.2 \mathrm{~Hz}$ ), 99.4, 94.3 (d, $J=3.4 \mathrm{~Hz}$ ), 86.9, 30.0, 28.8. LC-MS (ESI): $m / z=357.2[\mathrm{M}+\mathrm{H}]^{+}$. HRMS (ESI) $m / z:[\mathrm{M}+\mathrm{H}]^{+}$calculated for $\mathrm{C}_{22} \mathrm{H}_{17} \mathrm{FN}_{4}, 357.1510$; found 357.1515.

3-(Cyclopropylethynyl)- $N$,5-dimethyl-6-phenyl-5H-pyrrolo [2,3-b]pyrazin-2-amine (10k). LC-MS (ESI): $m / z=303.2[\mathrm{M}+\mathrm{H}]^{+}$. HRMS (ESI) $m / z$ : $[\mathrm{M}+\mathrm{H}]^{+}$calculated for $\mathrm{C}_{19} \mathrm{H}_{18} \mathrm{~N}_{4}, 303.1604$; found 303.1610 .

\subsection{General procedure for cyclization}

A suspension containing N,5-dimethyl-3-((trimethylsilyl) ethynyl)-5H-pyrrolo[2,3-b]pyrazin-2-amine $(0.125 \mathrm{~g}, 0.48 \mathrm{mmol})$ in DMF $(2 \mathrm{~mL})$ and $t$-BuOK $(0.1 \mathrm{~g}, 9.6 \mathrm{mmol})$ was stirred at $120{ }^{\circ} \mathrm{C}$ for $1 \mathrm{~h}$ under microwave. The reaction mixture was cooled to room temperature, diluted with EtOAc and water. The organic layer was separated, washed with water and dried over $\mathrm{MgSO}_{4}$, and concentrated in vacuum to give a pale yellow crystal which was subjected to silica gel column chromatography to give desired compound. 
1,5-Dimethyl-1,5-dihyrodipyrrolo[3,2-b:3', $2^{\prime}$-e $]$ pyrazine (11a). Mp: $192-193{ }^{\circ} \mathrm{C},{ }^{1} \mathrm{H}$ NMR $\left(500 \mathrm{MHz}, \mathrm{CDCl}_{3}\right) \delta 7.42(\mathrm{~d}, J=3.6 \mathrm{~Hz}$, $2 \mathrm{H}), 6.66$ (d, $J=3.6 \mathrm{~Hz}, 2 \mathrm{H}), 3.94(\mathrm{~s}, 6 \mathrm{H}) .{ }^{13} \mathrm{C}$ NMR $(126 \mathrm{MHz}$, $\left.\mathrm{CDCl}_{3}\right) \delta 139.6,134.9,132.0,98.7,31.6$. LC-MS (ESI): $m / z=187.1$ $[\mathrm{M}+\mathrm{H}]^{+}$. HRMS (ESI) $m / z:[\mathrm{M}+\mathrm{H}]^{+}$calculated for $\mathrm{C}_{10} \mathrm{H}_{10} \mathrm{~N}_{4}$, 187.0978; found 187.0988 .

1,5-Dimethyl-2-phenyl-1,5-dihyrodipyrrolo $\left[3,2-b: 3^{\prime}, 2^{\prime}-e\right] p y r-$ azine (11b). Mp: $156-157{ }^{\circ} \mathrm{C},{ }^{1} \mathrm{H} \mathrm{NMR}\left(500 \mathrm{MHz}, \mathrm{CDCl}_{3}\right) \delta 7.61$ $(\mathrm{d}, J=7.4 \mathrm{~Hz}, 2 \mathrm{H}), 7.51(\mathrm{t}, J=7.0 \mathrm{~Hz}, 2 \mathrm{H}), 7.47-7.43(\mathrm{~m}, 1 \mathrm{H})$, $7.41(\mathrm{~s}, 1 \mathrm{H}), 6.75(\mathrm{~s}, 1 \mathrm{H}), 6.68(\mathrm{~s}, 1 \mathrm{H}), 3.95(\mathrm{~s}, 3 \mathrm{H}), 3.94(\mathrm{~s}, 3 \mathrm{H})$. ${ }^{13} \mathrm{C}$ NMR $\left(126 \mathrm{MHz}, \mathrm{CDCl}_{3}\right) \delta 144.9,141.4,140.0,135.1,134.6$, 132.2, 131.7, 129.1, 128.6, 128.5, 99.1, 98.9, 31.7, 30.0. LC-MS (ESI): $m / z=263.1[\mathrm{M}+\mathrm{H}]^{+}$. HRMS (ESI) $m / z:[\mathrm{M}+\mathrm{H}]^{+}$calculated for $\mathrm{C}_{16} \mathrm{H}_{14} \mathrm{~N}_{4}, 263.1291$; found 263.1309.

2-(4-Fluorophenyl)-1,5-dimethyl-1,5-dihydrodipyrrolo[3,2b:3', $\mathbf{2}^{\prime}$-e]pyrazine (11c). ${ }^{1} \mathrm{H}$ NMR (500 $\left.\mathrm{MHz}, \mathrm{CDCl}_{3}\right) \delta$ 7.60-7.51 $(\mathrm{m}, 2 \mathrm{H}), 7.35(\mathrm{~d}, J=3.6 \mathrm{~Hz}, 1 \mathrm{H}), 7.23-7.12(\mathrm{~m}, 2 \mathrm{H}), 6.73(\mathrm{~s}, 1 \mathrm{H})$, $6.69(\mathrm{~d}, J=3.7 \mathrm{~Hz}, 1 \mathrm{H}), 3.92(\mathrm{~s}, 3 \mathrm{H}), 3.87$ (s, 3H). ${ }^{13} \mathrm{C}$ NMR $(126$ $\left.\mathrm{MHz}, \mathrm{CDCl}_{3}\right) \delta 162.8(\mathrm{~d}, J=248.8 \mathrm{~Hz}), 142.6,140.1,138.3,136.7$, 136.3, 130.9, 130.8, 130.7, 128.4 (d, $J=3.3 \mathrm{~Hz}), 115.7$ (d, $J=21.7$ $\mathrm{Hz}$ ), 99.8 (d, $J=15.8 \mathrm{~Hz}$ ), 31.3, 29.7. HRMS (ESI) $m / z:[\mathrm{M}+\mathrm{H}]^{+}$ calculated for $\mathrm{C}_{16} \mathrm{H}_{13} \mathrm{FN}_{4}, 281.1197$; found 284.1212.

4-(1,5-Dimethyl-1,5-dihydrodipyrrolo[3,2-b:3', $2^{\prime}$ - e ]pyrazin-2yl)- $\mathrm{N}, \boldsymbol{N}$-dimethylaniline (11d). Mp: $154-155{ }^{\circ} \mathrm{C},{ }^{1} \mathrm{H}$ NMR (500 $\left.\mathrm{MHz}, \mathrm{CDCl}_{3}\right) \delta 7.50(\mathrm{~d}, J=8.8 \mathrm{~Hz}, 2 \mathrm{H}), 7.36(\mathrm{~d}, J=3.6 \mathrm{~Hz}, 1 \mathrm{H})$, $6.83(\mathrm{~d}, J=8.8 \mathrm{~Hz}, 2 \mathrm{H}), 6.66(\mathrm{~d}, J=3.6 \mathrm{~Hz}, 1 \mathrm{H}), 6.65(\mathrm{~s}, 1 \mathrm{H}), 3.94$ $(\mathrm{d}, J=3.7 \mathrm{~Hz}, 6 \mathrm{H}), 3.04(\mathrm{~s}, 6 \mathrm{H}) .{ }^{13} \mathrm{C} \mathrm{NMR}\left(126 \mathrm{MHz}, \mathrm{CDCl}_{3}\right)$ $\delta$ 150.4, 146.1, 141.5, 139.8, 135.7, 133.8, 130.8, 130.0, 119.7, 112.0, 98.9, 97.5, 40.3, 31.7, 30.0. LC-MS (ESI): $m / z=306.2[\mathrm{M}+$ $\mathrm{H}]^{+}$. HRMS (ESI) $m / z$ : $[\mathrm{M}+\mathrm{H}]^{+}$calculated for $\mathrm{C}_{18} \mathrm{H}_{19} \mathrm{~N}_{5}$, 306.1713; found 306.1722.

4-(1,5-Dimethyl-1,5-dihydrodipyrrolo[3,2- $\left.b: 3^{\prime}, 2^{\prime}-e\right]$ pyrazin-2yl)benzonitrile (11e). Mp: $268-269{ }^{\circ} \mathrm{C},{ }^{1} \mathrm{H}$ NMR $(500 \mathrm{MHz}$, $\left.\mathrm{CDCl}_{3}\right) \delta 7.80(\mathrm{~d}, J=7.9 \mathrm{~Hz}, 2 \mathrm{H}), 7.73(\mathrm{~d}, J=8.0 \mathrm{~Hz}, 2 \mathrm{H}), 7.48(\mathrm{~d}$, $J=3.2 \mathrm{~Hz}, 1 \mathrm{H}), 6.84(\mathrm{~s}, 1 \mathrm{H}), 6.69(\mathrm{~d}, J=3.3 \mathrm{~Hz}, 1 \mathrm{H}), 3.97(\mathrm{~s}, 6 \mathrm{H})$. ${ }^{13} \mathrm{C}$ NMR $\left(126 \mathrm{MHz}, \mathrm{CDCl}_{3}\right) \delta 142.1,141.8,140.3,136.6,135.6$, 134.4, 132.9, 132.4, 129.4, 118.5, 111.9, 100.9, 99.0, 31.7, 30.3. LC-MS (ESI): $m / z=288[\mathrm{M}+\mathrm{H}]^{+}$. HRMS (ESI) $m / z:[\mathrm{M}+\mathrm{H}]^{+}$ calculated for $\mathrm{C}_{17} \mathrm{H}_{13} \mathrm{~N}_{5}, 288.1244$; found 288.1257.

1,5-Dimethyl-2,6-diphenyl-1,5-dihyrodipyrrolo $\left[3,2-b: 3^{\prime}, 2^{\prime}-e\right]$ pyrazine (11f). Mp: 258-260 ${ }^{\circ} \mathrm{C},{ }^{1} \mathrm{H} \mathrm{NMR}\left(500 \mathrm{MHz}, \mathrm{CDCl}_{3}\right.$ ) $\delta 7.67-7.63(\mathrm{~m}, 2 \mathrm{H}), 7.55(\mathrm{t}, J=7.5 \mathrm{~Hz}, 2 \mathrm{H}), 7.48(\mathrm{t}, J=7.4 \mathrm{~Hz}$, $1 \mathrm{H}), 6.80(\mathrm{~s}, 1 \mathrm{H}), 3.99(\mathrm{~s}, 3 \mathrm{H}) .{ }^{13} \mathrm{C} \mathrm{NMR}\left(126 \mathrm{MHz}, \mathrm{CDCl}_{3}\right)$ $\delta$ 144.7, 141.8, 134.9, 132.2, 129.1, 128.7, 128.5, 99.3, 30.1; LCMS (ESI): $m / z=339.2[\mathrm{M}+\mathrm{H}]^{+}$. HRMS (ESI) $m / z:[\mathrm{M}+\mathrm{H}]^{+}$ calculated for $\mathrm{C}_{22} \mathrm{H}_{18} \mathrm{~N}_{4}, 339.1604$; found 339.1610.

2-(4-Methoxyphenyl)-1,5-dimethyl-6-phenyl-1,5-dihydrodipyrrolo[3,2-b:3', $\mathbf{2}^{\prime}$-e]pyrazine (11g). Mp: $228-230{ }^{\circ} \mathrm{C} .{ }^{1} \mathrm{H}$ NMR $\left(500 \mathrm{MHz} \mathrm{CDCl}_{3}\right) \delta 7.65(\mathrm{~d}, J=7.2 \mathrm{~Hz}, 2 \mathrm{H}), 7.60-7.52(\mathrm{~m}, 4 \mathrm{H})$, $7.47(\mathrm{t}, J=7.4 \mathrm{~Hz}, 1 \mathrm{H}), 7.07(\mathrm{~d}, J=8.6 \mathrm{~Hz}, 2 \mathrm{H}), 6.79(\mathrm{~s}, 1 \mathrm{H})$, $6.73(\mathrm{~s}, 1 \mathrm{H}), 3.98(\mathrm{~s}, 3 \mathrm{H}), 3.96(\mathrm{~s}, 3 \mathrm{H}), 3.91(\mathrm{~s}, 3 \mathrm{H}) .{ }^{13} \mathrm{C}$ NMR $\left(126 \mathrm{MHz}, \mathrm{CDCl}_{3}\right) \delta 159.9,144.7,144.3,141.7,135.1,134.5$, $132.3,130.4,129.1,128.7,128.4,124.6,114.7,114.2$, 99.3, 98.6, 55.4, 30.1, 30.0. LC-MS (ESI): $m / z=369.2[\mathrm{M}+\mathrm{H}]^{+}$. HRMS (ESI) $m / z:[\mathrm{M}+\mathrm{H}]^{+}$calculated for $\mathrm{C}_{23} \mathrm{H}_{20} \mathrm{~N}_{5} \mathrm{O}, 369.1710$; found 369.1717 .
1,5-Dimethyl-2-phenyl-6-p-tolyl-1,5-dihydrodipyrrolo[3,2b:3', $\mathbf{2}^{\prime}$-e]pyrazine (11h). Mp: 204-206 ${ }^{\circ} \mathrm{C} .{ }^{1} \mathrm{H}$ NMR $(500 \mathrm{MHz}$, $\left.\mathrm{CDCl}_{3}\right) \delta 7.67-7.64(\mathrm{~m}, 2 \mathrm{H}), 7.56-7.53(\mathrm{~m}, 4 \mathrm{H}), 7.48(\mathrm{~d}, J=$ $7.4 \mathrm{~Hz}, 1 \mathrm{H}), 7.36(\mathrm{~d}, J=7.9 \mathrm{~Hz}, 2 \mathrm{H}), 6.79(\mathrm{~s}, 1 \mathrm{H}), 6.76(\mathrm{~s}, 1 \mathrm{H})$, 3.99 (s, 3H), 3.98 (s, 3H), 2.48 (s, 3H). ${ }^{13} \mathrm{C} \mathrm{NMR}\left(126 \mathrm{MHz}, \mathrm{CDCl}_{3}\right)$ $\delta 144.9,144.4,141.8,141.8,138.6,135.0,134.7,132.3,129.4$, 129.3, 129.1, 129.0, 128.7, 128.5, 99.3, 98.9, 30.15, 30.1, 21.3. LCMS (ESI): $m / z=353.3[\mathrm{M}+\mathrm{H}]^{+}$. HRMS (ESI) $m / z:[\mathrm{M}+\mathrm{H}]^{+}$ calculated for $\mathrm{C}_{23} \mathrm{H}_{20} \mathrm{~N}_{4}, 353.1761$; found 353.1774.

4-(1,5-Dimethyl-6-phenyl-1,5-dihydrodipyrrolo $\left[3,2-b: 3^{\prime}, 2^{\prime}-e\right]$ pyrazin-2-yl)- $\boldsymbol{N}, \boldsymbol{N}$-dimethylaniline (11i). Mp: $219-220{ }^{\circ} \mathrm{C} .{ }^{1} \mathrm{H}$ NMR $\left(500 \mathrm{MHz}, \mathrm{CDCl}_{3}\right) \delta 7.65$ (d, $\left.J=7.2 \mathrm{~Hz}, 2 \mathrm{H}\right), 7.54$ (dd, $J=$ $8.2,4.9 \mathrm{~Hz}, 4 \mathrm{H}), 7.47$ (d, $J=7.4 \mathrm{~Hz}, 1 \mathrm{H}), 6.87(\mathrm{~d}, J=8.7 \mathrm{~Hz}, 2 \mathrm{H})$, $6.79(\mathrm{~s}, 1 \mathrm{H}), 6.70(\mathrm{~s}, 1 \mathrm{H}), 3.98(\mathrm{~s}, 6 \mathrm{H}), 3.08(\mathrm{~s}, 6 \mathrm{H}) .{ }^{13} \mathrm{C}$ NMR $(126$ $\left.\mathrm{MHz}, \mathrm{CDCl}_{3}\right) \delta 150.4,145.9,143.7,141.9,141.7,135.5,134.1$, 132.4, 130.0, 129.1, 128.7, 128.3, 119.7, 112.1, 99.3, 97.7, 40.3, 30.1, 30.1. LC-MS (ESI): $m / z=382.3[\mathrm{M}+\mathrm{H}]^{+}$. HRMS (ESI) $m / z$ : $[\mathrm{M}+\mathrm{H}]^{+}$calculated for $\mathrm{C}_{24} \mathrm{H}_{23} \mathrm{~N}_{5}, 382.2026$; found 382.2035.

2-(3-Fluorophenyl)-1,5-dimethyl-6-phenyl-1,5-dihydrodipyrrolo[3,2-b:3', $\left.2^{\prime}-e\right]$ pyrazine (11j). Mp: $234-236{ }^{\circ} \mathrm{C} .{ }^{1} \mathrm{H}$ NMR (500 $\left.\mathrm{MHz}, \mathrm{CDCl}_{3}\right) \delta 7.67-7.64(\mathrm{~m}, 2 \mathrm{H}), 7.55(\mathrm{t}, J=7.5 \mathrm{~Hz}, 2 \mathrm{H}), 7.53-$ $7.47(\mathrm{~m}, 2 \mathrm{H}), 7.44(\mathrm{~d}, J=7.7 \mathrm{~Hz}, 1 \mathrm{H}), 7.36(\mathrm{dd}, J=9.4,1.9 \mathrm{~Hz}$, $1 \mathrm{H}), 7.18(\mathrm{~d}, J=1.7 \mathrm{~Hz}, 1 \mathrm{H}), 6.81(\mathrm{~s}, 1 \mathrm{H}), 6.80(\mathrm{~s}, 1 \mathrm{H}), 4.00(\mathrm{~s}$, 3H), 3.99 (s, 3H). ${ }^{13} \mathrm{C}$ NMR (126 MHz, $\left.\mathrm{CDCl}_{3}\right) \delta 162.7(\mathrm{~d}, J=$ $246.8 \mathrm{~Hz}$ ), 145.1, 143.0 (d, $J=2.4 \mathrm{~Hz}), 141.9$ (d, $J=10.2 \mathrm{~Hz}$ ), 135.3, 134.5, 134.3 (d, $J=8.2 \mathrm{~Hz}$ ), 132.1, 130.3, 130.3, 129.1, 128.7, 128.6, 124.8 (d, $J=2.9 \mathrm{~Hz}), 115.9(\mathrm{~d}, J=22.4 \mathrm{~Hz}), 115.4(\mathrm{~d}$, $J=21.1 \mathrm{~Hz}$ ), 99.9, 99.3, 30.2, 30.1. LC-MS (ESI): $m / z=357.3[\mathrm{M}+$ $\mathrm{H}]^{+}$. HRMS (ESI) $m / z$ : $[\mathrm{M}+\mathrm{H}]^{+}$calculated for $\mathrm{C}_{22} \mathrm{H}_{17} \mathrm{FN}_{4}$, 357.1510; found 357.1527.

2-Cyclopropyl-1,5-dimethyl-6-phenyl-1,5-dihydrodipyrrolo [3,2-b:3 $\left.\mathbf{3}^{\prime}, \mathbf{2}^{\prime}-\boldsymbol{e}\right]$ pyrazine (11k). Mp: $120-121^{\circ} \mathrm{C} .{ }^{1} \mathrm{H} \mathrm{NMR}(500 \mathrm{MHz}$, $\left.\mathrm{CDCl}_{3}\right) \delta 7.62-7.58(\mathrm{~m}, 2 \mathrm{H}), 7.49(\mathrm{dd}, J=10.7,4.2 \mathrm{~Hz}, 2 \mathrm{H}), 7.42$ $(\mathrm{td}, J=7.1,1.1 \mathrm{~Hz}, 1 \mathrm{H}), 6.74(\mathrm{~s}, 1 \mathrm{H}), 6.26(\mathrm{~s}, 1 \mathrm{H}), 3.97(\mathrm{~d}, J=$ $1.3 \mathrm{~Hz}, 3 \mathrm{H}), 3.93(\mathrm{~d}, J=0.8 \mathrm{~Hz}, 3 \mathrm{H}), 2.03-1.96(\mathrm{~m}, 1 \mathrm{H}), 1.09$ (d, $J$ $=8.2 \mathrm{~Hz}, 2 \mathrm{H}), 0.86(\mathrm{~d}, J=5.1 \mathrm{~Hz}, 2 \mathrm{H}) .{ }^{13} \mathrm{C} \mathrm{NMR}(126 \mathrm{MHz}$, $\left.\mathrm{CDCl}_{3}\right) \delta 147.6,143.5,141.4,140.9,135.0,133.8,132.4,129.1$, 128.6, 128.2 99.2, 94.6, 30.0, 28.2, 8.2, 7.1. LC-MS (ESI): $m / z=$ $303.3[\mathrm{M}+\mathrm{H}]^{+}$. HRMS (ESI) $m / z:[\mathrm{M}+\mathrm{H}]^{+}$calculated for $\mathrm{C}_{19} \mathrm{H}_{18} \mathrm{~N}_{4}, 303.1604$; found 303.1620.

3-Chloro-5-methyl-2-(phenylethynyl)-5H-pyrrolo[2,3-b]pyrazine (12b). The procedure described above for 5 a was used. The titled compound was obtained as pale yellow solid with $72 \%$ yield. LC-MS (ESI): $m / z=344.1[\mathrm{M}+\mathrm{H}]^{+}$. HRMS (ESI) $m / z:[\mathrm{M}+$ $\mathrm{H}]^{+}$calculated for $\mathrm{C}_{21} \mathrm{H}_{14} \mathrm{ClN}_{4}, 344.0949$; found 344.0954.

\subsection{General procedure for preparing pyrrolothienopyrazine}

To a solution of 3-chloro-5-methyl-2-(phenylethynyl)-5 $H$-pyrrolo $[2,3-b]$ pyrazine $(0.12 \mathrm{~g} 0.4 \mathrm{mmol})$ in DMF $(4 \mathrm{~mL})$ was added $\mathrm{Na}_{2} \mathrm{~S} \cdot 5 \mathrm{H}_{2} \mathrm{O}(0.14 \mathrm{~g}, 1.2 \mathrm{mmol})$. The mixture was refluxed for $1 \mathrm{~h}$, cooled to ambient temperature then diluted with EtOAc and water. The organic layer was separated, dried over $\mathrm{MgSO}_{4}$, and concentrated in vacuum to give a residue that was subjected to silica gel column chromatography to give a desired compound.

7-Methyl-2-phenyl-7H-pyrrolo[3,2-e]thieno[2,3-b]pyrazine (13a). Yield: $74 \%$, light brown solid. Mp: $196-197{ }^{\circ} \mathrm{C},{ }^{1} \mathrm{H}$ NMR $(500 \mathrm{MHz}$, 
$\left.\mathrm{CDCl}_{3}\right) \delta 7.82-7.78(\mathrm{~m}, 2 \mathrm{H}), 7.75(\mathrm{~s}, 1 \mathrm{H}), 7.52(\mathrm{~d}, J=3.6 \mathrm{~Hz}, 1 \mathrm{H})$, $7.49(\mathrm{t}, J=7.6 \mathrm{~Hz}, 2 \mathrm{H}), 7.42(\mathrm{~d}, J=7.3 \mathrm{~Hz}, 1 \mathrm{H}), 6.74(\mathrm{~d}, J=3.6 \mathrm{~Hz}$, 1H), 3.97 (s, 3H). ${ }^{13} \mathrm{C}$ NMR (126 MHz, $\left.\mathrm{CDCl}_{3}\right) \delta$ 149.9, 146.3, 144.1, 139.4, 138.9, 134.1, 133.9, 129.1, 128.9, 126.3, 117.8, 100.3, 31.6. LC-MS (ESI): $m / z=266.1[\mathrm{M}+\mathrm{H}]^{+}$. HRMS (ESI) $m / z:[\mathrm{M}+\mathrm{H}]^{+}$ calculated for $\mathrm{C}_{15} \mathrm{H}_{11} \mathrm{~N}_{4} \mathrm{~S}, 266.0746$; found 266.0751.

7-Methyl-2,6-phenyl-7H-pyrrolo[3,2-e]thieno[2,3-b]pyrazine (13b). Yield: 69\%, light brown solid. Mp: 194-196 ${ }^{\circ} \mathrm{C},{ }^{1} \mathrm{H}$ NMR $\left(500 \mathrm{MHz}, \mathrm{CDCl}_{3}\right) \delta 7.78(\mathrm{~d}, J=7.5 \mathrm{~Hz}, 2 \mathrm{H}), 7.74(\mathrm{~s}, 1 \mathrm{H}), 7.63-$ $7.60(\mathrm{~m}, 2 \mathrm{H}), 7.54(\mathrm{t}, J=7.3 \mathrm{~Hz}, 2 \mathrm{H}), 7.50-7.45(\mathrm{~m}, 3 \mathrm{H}), 7.39(\mathrm{t}, J$ $=7.4 \mathrm{~Hz}, 1 \mathrm{H}), 6.78(\mathrm{~s}, 1 \mathrm{H}), 3.93(\mathrm{~s}, 3 \mathrm{H}) .{ }^{13} \mathrm{C} \mathrm{NMR}(126 \mathrm{MHz}$, $\left.\mathrm{CDCl}_{3}\right) \delta 149.5,146.9,146.7,143.9,141.2,139.1,134.1,131.4$, 129.1, 129.1, 129.0, 128.8, 128.8, 126.2, 117.8, 100.1, 30.0. LC-MS (ESI): $m / z=342.2[\mathrm{M}+\mathrm{H}]^{+}$. HRMS (ESI) $m / z:[\mathrm{M}+\mathrm{H}]^{+}$calculated for $\mathrm{C}_{21} \mathrm{H}_{16} \mathrm{~N}_{4} \mathrm{~S}$, 342.1059; found 342.1068.

5-Bromo-3,6-dichloropyrazine-2-amine (15). Yield: 90\%, pale yellow solid. Mp: $129-130{ }^{\circ} \mathrm{C},{ }^{1} \mathrm{H}$ NMR $\left(500 \mathrm{MHz}, \mathrm{CDCl}_{3}\right) \delta 5.24$ (s, 2H). ${ }^{13} \mathrm{C} \mathrm{NMR}\left(126 \mathrm{MHz}, \mathrm{CDCl}_{3}\right) \delta 149.7,145.5,129.4,121.5$. LC-MS (ESI): $m / z=339.9[\mathrm{M}-1]^{-}$.

2,5-Dibromo-3,6-dichloropyrazine (16). To a solution of 5-bromo-3,6-dichloropyrazin-2-amine $(0.1 \mathrm{~g}, 0.4 \mathrm{mmol})$ in THF $(1 \mathrm{~mL}), \mathrm{HBr}(2 \mathrm{~mL})$ at $0{ }^{\circ} \mathrm{C}$ was added $\mathrm{NaNO}_{2}(0.07 \mathrm{~g}, 1.0 \mathrm{mmol})$ slowly. The mixture was stirred at $0{ }^{\circ} \mathrm{C}$ for $20 \mathrm{~min}$ and then made basic by using $1 \mathrm{M} \mathrm{NaOH}$ and extracted with EtOAc $(3 \times)$. The combined organic layers were dried over $\mathrm{MgSO}_{4}$, and concentrated to give a residue that was subjected to silica gel column chromatography to give desired compound as pale a yellow solid. Yield: 77\%, mp: $93-94{ }^{\circ} \mathrm{C},{ }^{13} \mathrm{C}$ NMR $(126 \mathrm{MHz}$, $\left.\mathrm{CDCl}_{3}\right) \delta$ 146.6, 136.3.

2,5-Dichloro-3,6-bis(phenylethynyl)pyrazine (17). To a stirred solution of 2,5-dibromo-3,6-dichloropyrazine (0.6 g, 1.97 $\mathrm{mmol})$ in THF $(10 \mathrm{~mL})$, then TEA $(1.3 \mathrm{~mL}, 13 \mathrm{mmol})$, $\mathrm{Pd}\left(\mathrm{PPh}_{3}\right)_{2} \mathrm{Cl}_{2}(0.27 \mathrm{~g}, 0.34 \mathrm{mmol})$ and $\mathrm{CuI}(75 \mathrm{mg}, 0.34 \mathrm{mmol})$ were added subsequently under nitrogen atmosphere. After $10 \mathrm{~min}$, phenyl acetylene $(0.42 \mathrm{~g}, 4 \mathrm{mmol})$ was added drop wise and the mixture was stirred at room temperature for $1.5 \mathrm{~h}$. The mixture was concentrated in a vacuum to give a residue that was diluted with water and extracted with EtOAc $(2 \times)$. The combined organic layer was washed with water and dried over $\mathrm{MgSO}_{4}$, and concentrated in vacuum to give a residue that was subjected to silica gel column chromatography to yield the desired compound as pale yellow solid. Yield 38\%, ${ }^{1} \mathrm{H}$ NMR $(500$ $\left.\mathrm{MHz} \mathrm{CDCl}_{3}\right) \delta 7.66(\mathrm{~d}, J=7.0 \mathrm{~Hz}, 4 \mathrm{H}), 7.43(\mathrm{dt}, J=14.5,7.1 \mathrm{~Hz}$, $6 \mathrm{H}) .{ }^{13} \mathrm{C}$ NMR $\left(126 \mathrm{MHz}, \mathrm{CDCl}_{3}\right) \delta 147.5,135.8,132.4,130.4$, 128.6, 120.9, 100.3, 84.3. LC-MS (ESI): $m / z=349.0[\mathrm{M}+\mathrm{H}]^{+}$.

\section{Conclusions}

In the study described above, we developed a convenient method for the synthesis of 1,5- and 1,7-dihydrodipyrrolo[2,3$b ; 3^{\prime} 2^{\prime}$-e]pyrazine derivatives. Protocols were devised to generate either 2-amino- or 3-amino-pyrrolopyrazines from the corresponding 2-bromo-3-chloro-5H-pyrrolo[2,3- $b]$ pyrazines. Specifically, amination reactions of the dihalo substrates carried under metal free conditions in the presence of methylamine under MW irradiation produce 3-amino-pyrrolopyrazine exclusively. In contrast, Buchwald cross coupling affords 2-amino- pyrrolopyrazine. The pyrrolo pyrazine scaffolds were converted to the respective 1,7- and 1,5-DPP derivatives using the Sonogashira coupling reactions. All the synthesized compounds were found to be highly crystalline and readily soluble in organic solvents. Furthermore, the prepared pyrazine derivatives have high thermal stabilities, suggesting that they have high potential for use in organic electronic devices. Ongoing research in our laboratory is aimed at exploring interesting features of the 1,5-dihydrodipyrrolo[3,2- $\left.b: 3^{\prime}, 2^{\prime}-e\right]$ pyrazine molecular scaffold.

\section{Acknowledgements}

This research was supported by Basic Science Research Program through the National Research Foundation of Korea (NRF) funded by the Ministry of Education (No. 2016R1D1A1B04932654), and Sabbatical Research Program of the Dongguk University in Seoul, Korea.

\section{References}

1 J. Zhang, J. Wang, X. Xu, S. Chen, Q. Zhang, C. Yao, X. Zhuang, A. Pan and L. Li, J. Mater. Chem. C, 2015, 3, 5933-5939.

2 Z. Liang, Q. Tang, R. Mao, D. Liu, J. Xu and Q. Miao, Adv. Mater., 2011, 23, 5514-5518.

3 K. Liu, C.-L. Song, Y.-C. Zhou, X.-Y. Zhou, X.-J. Pan, L.-Y. Cao, C. Zhang, Y. Liu, X. Gong and H.-L. Zhang, J. Mater. Chem. C, 2015, 3, 4188-4196.

4 Y.-I. Park, J.-H. Son, J.-S. Kang, S.-K. Kim, J.-H. Lee and J.-W. Park, Chem. Commun., 2008, 2143-2145, DOI: 10.1039/b718873k.

5 L. Yan, D. Zhao, J. Lan, Y. Cheng, Q. Guo, X. Li, N. Wu and J. You, Org. Biomol. Chem., 2013, 11, 7966-7977.

6 P. Henriksson, C. Lindqvist, B. Abdisa, E. Wang, Z. George, R. Kroon, C. Müller, T. Yohannes, O. Inganäs and M. R. Andersson, Sol. Energy Mater. Sol. Cells, 2014, 130, 138-143.

7 X. Lu, G. Zhou, H. Wang, Q. Feng and Z.-S. Wang, Phys. Chem. Chem. Phys., 2012, 14, 4802-4809.

8 C. R. Hopkins and N. Collar, Tetrahedron Lett., 2004, 45, 8087-8090.

9 C. R. Hopkins and N. Collar, Tetrahedron Lett., 2004, 45, 8631-8633.

10 I. Simpson, S. A. St-Gallay, S. Stokes, D. T. E. Whittaker and R. Wiewiora, Tetrahedron Lett., 2015, 56, 1492-1495.

11 A. Keivanloo, M. Bakherad, H. Nasr-Isfahani and S. Esmaily, Tetrahedron Lett., 2012, 53, 3126-3130.

12 G. Bashiardes, I. Safir, A. S. Mohamed, F. Barbot and J. Laduranty, Org. Lett., 2003, 5, 4915-4918.

13 G. Xu and Y.-G. Wang, Org. Lett., 2004, 6, 985-987.

14 D. R. Sauer, D. Kalvin and K. M. Phelan, Org. Lett., 2003, 5, 4721-4724.

15 L. Shi, M. Wang, C.-A. Fan, F.-M. Zhang and Y.-Q. Tu, Org. Lett., 2003, 5, 3515-3517.

16 F. Y. Kwong and S. L. Buchwald, Org. Lett., 2003, 5, 793796. 
17 C. Bathula, C. E. Song, S. Badgujar, S.-J. Hong, I.-N. Kang, S.-J. Moon, J. Lee, S. Cho, H.-K. Shim and S. K. Lee, J. Mater. Chem., 2012, 22, 22224-22232.

18 E. Zhu, G. Ge, J. Shu, M. Yi, L. Bian, J. Hai, J. Yu, Y. Liu, J. Zhou and W. Tang, J. Mater. Chem. A, 2014, 2, 1358013586.

19 Q. Meng, L. Jiang, Z. Wei, C. Wang, H. Zhao, H. Li, W. Xu and W. Hu, J. Mater. Chem., 2010, 20, 10931-10935.

20 H. Wang, Y. Xu, X. Yu, R. Xing, J. Liu and Y. Han, Polymers, 2013, 5, 1272.

21 H. Dong, C. Wang and W. Hu, Chem. Commun., 2010, 46, 5211-5222.

22 C. Bathula, M. Kim, C. E. Song, W. S. Shin, D.-H. Hwang, J.-C. Lee, I.-N. Kang, S. K. Lee and T. Park, Macromolecules, 2015, 48, 3509-3515.
23 Y. Geng, J. Wang, S. Wu, H. Li, F. Yu, G. Yang, H. Gao and Z. Su, J. Mater. Chem., 2011, 21, 134-143.

24 Z. Liang, Q. Tang, J. Liu, J. Li, F. Yan and Q. Miao, Chem. Mater., 2010, 22, 6438-6443.

25 S. Van Epps, B. Fiamengo, J. Edmunds, A. Ericsson, K. Frank, M. Friedman, D. George, J. George, E. Goedken, B. Kotecki, G. Martinez, P. Merta, M. Morytko, S. Shekhar, B. Skinner, K. Stewart, J. Voss, G. Wallace, L. Wang, L. Wang and N. Wishart, Bioorg. Med. Chem. Lett., 2013, 23, 693-698.

26 S. K. Kolli, A. Nakhi, R. Medishetti, S. Yellanki, P. Kulkarni, R. Ramesh Raju and M. Pal, Bioorg. Med. Chem. Lett., 2014, 24, 4460-4465.

27 CCDC deposit number 1536706. 